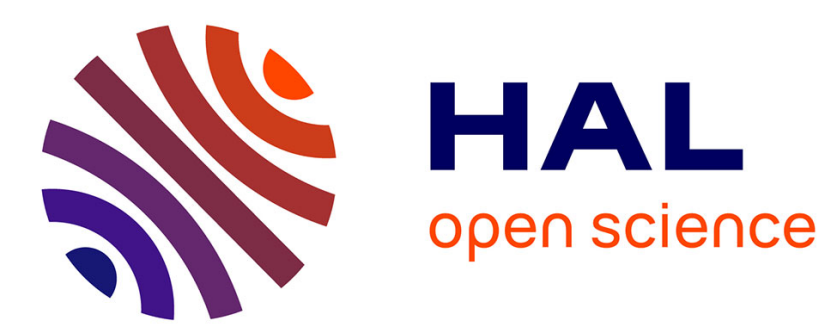

\title{
Benefits of Local Cooperation in Sectorized Cellular Networks under a Complexity Constraint
}

\author{
Samet Gelincik, Michele Wigger, Ligong Wang
}

\section{To cite this version:}

Samet Gelincik, Michele Wigger, Ligong Wang. Benefits of Local Cooperation in Sectorized Cellular Networks under a Complexity Constraint. IEEE Transactions on Wireless Communications, 2021, 20 (6), pp.3897-3910. 10.1109/TWC.2021.3054337 . hal-02940437

\section{HAL Id: hal-02940437 https://hal.science/hal-02940437}

Submitted on 16 Sep 2020

HAL is a multi-disciplinary open access archive for the deposit and dissemination of scientific research documents, whether they are published or not. The documents may come from teaching and research institutions in France or abroad, or from public or private research centers.
L'archive ouverte pluridisciplinaire HAL, est destinée au dépôt et à la diffusion de documents scientifiques de niveau recherche, publiés ou non, émanant des établissements d'enseignement et de recherche français ou étrangers, des laboratoires publics ou privés. 


\title{
Benefits of Local Cooperation in Sectorized
}

\section{Cellular Networks under a Complexity}

\section{Constraint}

\author{
Samet Gelincik Student Member, IEEE, Michèle Wigger, Senior Member, IEEE, \\ and Ligong Wang, Member, IEEE
}

\begin{abstract}
The paper presents upper and lower bounds on the degrees of freedom (DoF) of a sectorized hexagonal cellular model when neighboring base stations (BSs) can cooperate during at most $\kappa$ interaction rounds over rate-limited backhaul links. The lower bound is based on practically implementable beamforming and adapts the way BSs cooperate to the sectorization of the cells. It improves over the naive approach that ignores this sectorization in terms of the sum-rate, both at finite signal-to-noise ratio (SNR) and in the high-SNR limit. For moderate SNR, the new scheme improves also over an opportunistic cooperation strategy where each message is decoded based on the signals received at the three adjacent sectors with the best SNR. The upper bound is information-theoretic and holds for all possible coding schemes, including for example ergodic interference alignment whose practical implementation currently seems out of reach. Lower and upper bounds show that the complexity constraint, imposed by limiting the number of interaction rounds $\kappa$, indeed limits the largest achievable sum-rate and DoF. In particular, irrespective of the backhaul capacity $\mu$, the per-user DoF cannot exceed a threshold which depends on $\kappa$.
\end{abstract}

S. Gelincik and M. Wigger are with the Communictations and Electronics Department, LTCI, Telecom Paris, 75013 Paris, France.

Ligong Wang is with ETIS Laboratory-Unitersité Paris Seine, Université de Cergy-Pontoise, ENSEA, CNRS.

The work of M. Wigger was supported by Huawei Inc. Grant Agreement YB2015120036.

This works was presented in part at the 15th International Symposium on Wireless Communication Systems, Lisbon, Portugal, 28-31 Aug. 2018, see [7]. 


\section{INTRODUCTION}

This paper considers the uplink of a hexagonal cellular network with mobile users and base stations (BSs) that are equipped with multiple directional antennas, which allow them to create intracell sectors whose communications do not interfere. Following current standards, each cell is assumed to be divided into three sectors, and each BS to have the same number $M$ of antennas pointing at any of the cell's sectors. The main quantity of interest in this paper are the sumcapacity and the degrees of freedom (DoF) per user. The DoF per-user, in the following called DoF, of any generic [8] interference network with $M$-antenna transmitters and receivers is $M / 2$ [8]. Achieving this DoF however requires interference alignment techniques that currently cannot be implemented in practical systems.

DoF $M / 2$ can also be achieved with more practical coding techniques such as one-shot interference alignment if the BSs can cooperate over backhaul links [15] which allow them to successively cancel part of the interference. However, such a successive interference cancellation scheme introduces long decoding delays, thus limiting its practical implementations. To avoid such long delays, in this paper we constrain the number of interaction rounds between BSs, which prevents the BSs to run very long cooperation protocols as required in the repeated successive interference cancellation schemes in [15].

Cooperation between communication devices (BSs or mobiles) has first been considered in [23], and subsequently in many other works such as [1], [3], [4], [12], [16]-[22]. In the context of cellular systems, cooperation has mostly been used to create alternate communication paths (by having mobile users or dedicated terminals relay the transmit signals of adjacent mobiles, see e.g., [2], [5], [10], [13]), or to provide BSs with quantized versions of the signals received at other BSs (allowing for clustered decoding, see e.g., [9], [11], [14], [16], [19], [20]). In this work, we consider cooperation beetween BSs and we propose a new coding scheme based on the idea of decomposing the network into clusters combined with clustered decoding. The main contribution of our scheme is the specific way we build the clusters. One could be tempted to deactivate entire cells. We find a way to decompose the network into separate clusters by only deactivating the users in single sectors of certain cells but not the entire cells. Hereby we satisfy a given complexity constraint on the backhaul cooperation that restricts the number of interaction rounds, and as such the size of the clusters. The imposed complexity constraint is not active for various small networks, were few interaction rounds suffice, see e.g., [4], [12], [22], 
[23]. However, for large networks it has been shown that it can be beneficial if the number of interaction rounds grows with the number of users [21]. If this is not possible, the performance of the communication deteriorates.

The main interest of this paper is to understand the maximum sum-rate and DoF that are achievable on the uplink of a sectorized hexagonal model when the backhaul cooperation between BSs is limited in capacity and in the number of interaction rounds. We provide upper and lower bounds on the maximum DoF and a lower bound on the maximum sum-rate. Our new lower bounds are based on the coding scheme described above and show that DoF $M / 2$ is achievable in the sectorized hexagonal cellular model without interference alignment and with only two BS-interaction rounds and a backhaul DoF of $M / 6$. Notice that a naive approach that builds the clusters by deactivating entire cells would need $M / 3$ backhaul DoF to achieve $M / 2$ DoF in the transmission from the mobile users to the BSs. Generally, the new lower bound improves over this naive approach in all parameter ranges.

The upper bound presented in this paper is information-theoretic and allows for any coding scheme. (In particular, it also includes other cooperation strategies beyond clustered decoding.) The proposed upper bound shows that for small backhaul capacities, the DoF of the proposed scheme is close to optimal, and that irrespectively of the capacities of the backhaul links, for finite $\kappa$, the DoF is bounded away from $M$ when the number of cells grows large.

\section{PRoblem Definition}

\section{A. Network Model}

Consider the uplink communication of a cellular network consisting of $N \gg 1$ hexagonal cells as depicted in Figure 1. Each cell contains a base station (BS) equipped with $3 M$ directional antennas, where a third of these antennas covers each of the three sectors of the cell. Usage of directional antennas, where side lobe radiation patterns are negligible, implies that communications in the three sectors of a cell do not interfere each other. This leads to the interference graph in Figure 1, where each sector is depicted by a small circle, and interfering sectors in different cells are connected by solid black lines, whereas noninterfering sectors in the same cell are not connected.

Mobile users in a sector perform orthogonal multiple-access as is typical for current networks, and we thus restrict our model to a single mobile user per sector. For simplicity and symmetry, 


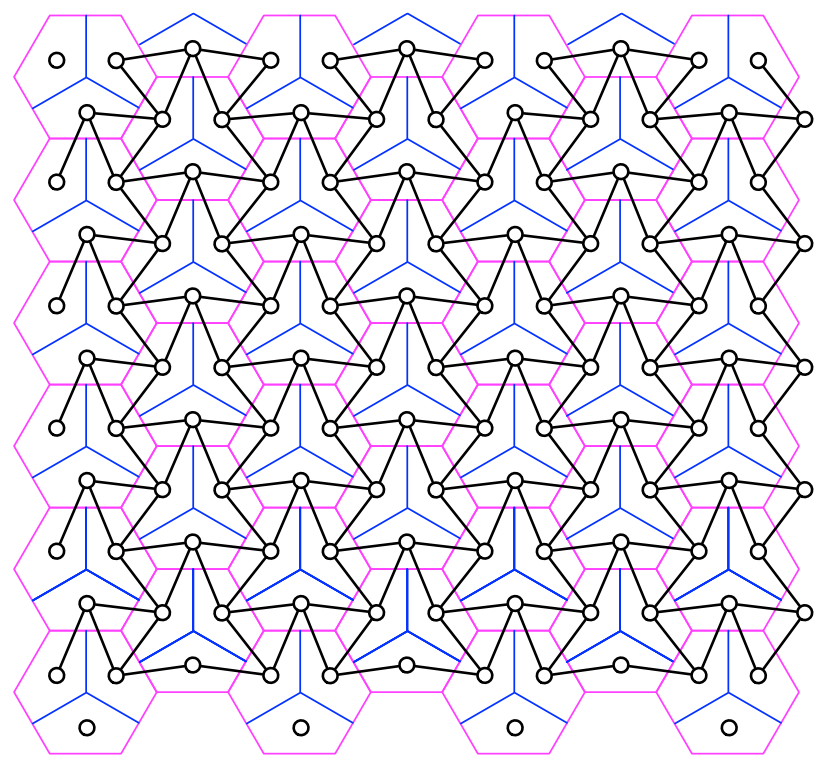

Fig. 1: Sectorized cellular model. Purple hexagonal regions depict the various cells and the blue lines divide them into sectors. Each circle depicts a mobile-user that is equipped with $M$ transmit antennas. Each cell is associated to a BS, which for each of the three sectors has a set of $M$ receive antennas directed to that sector.

we assume that this mobile user is equipped with $M$ antennas. The uplink communication is then modeled by the following discrete-time input-output relation:

$$
\mathbf{y}_{u, t}=\mathbf{H}_{u, u} \mathbf{x}_{u, t}+\sum_{\substack{v: \text { sector } v \\ \text { interferes sector } u}} \mathbf{H}_{u, v} \mathbf{x}_{v, t}+\mathbf{z}_{u, t}, \quad t \in\{1, \ldots, n\}
$$

where

- $u \in\{1, \ldots, 3 N\}$ denotes the index of a given cell-sector;

- $\mathbf{x}_{u, t}$ denotes the $M$-dimensional time- $t$ signal sent by mobile user $u$;

- $\mathbf{y}_{u, t}$ denotes the $M$-dimensional time- $t$ signal received at the $M$ BS receive antennas directing to sector $u$;

- $\mathbf{z}_{u, t}$ denotes the $M$-dimensional independent and identically distributed (i.i.d.) standard Gaussian noise vector corrupting the time-t signal at user $u$, which is independent of all other noise vectors; 
- and $\mathbf{H}_{u^{\prime}, u}$ denotes an $M$-by- $M$ random matrix that models the channel from mobile user $u$ to the receive-antennas in sector $u^{\prime}$.

Channel matrices are assumed to be constant during the entire transmission and known to all transmitters and receivers. We shall further specify these channel matrices a bit later in the paper.

\section{B. Communication Model with Backhaul Cooperation}

Consider the uplink communication over the cellular network described in the previous section where each mobile user $u \in\{1, \ldots, 3 N\}$ wishes to send an independent message $W_{u}$, which is uniformly distributed over $\left\{1, \ldots,\left\lfloor 2^{n R_{u}}\right\rfloor\right\}$, to the single BS in its cell. Communication takes place in two phases. In the first phase, each mobile user $u$ applies an encoding function $f_{u}^{(n)}:\left\{1, \ldots,\left\lfloor 2^{n R_{u}}\right\rfloor\right\} \rightarrow \mathbb{R}^{M \times n}$ to its message $W_{u}$ and sends the $n$ resulting vectors

$$
\mathbf{X}_{u}:=\left(\mathbf{X}_{u, 1}, \ldots, \mathbf{X}_{u, n}\right)=f_{u}^{(n)}\left(W_{u}\right)
$$

as its inputs over the network. Each encoding function $f_{u}^{(n)}$ has to be chosen so that the input vectors satisfy an average input power constraint $P$ :

$$
\frac{1}{n} \sum_{t=1}^{n}\left\|\mathbf{X}_{u, t}\right\|^{2} \leq P \quad \text { with probability } 1 .
$$

The subsequent second communication phase takes place after each receiving BS $j \in\{1, \ldots, N\}$ has observed all outputs at the antennas in each of the three sectors of the cell. The second phase takes place over the backhaul links connecting adjacent BSs. It is assumed to be noise-free but rate-limited and can be interactive. However, to limit complexity and latency of the backhaul communications, we constrain the interaction to at most $\kappa$ rounds, for a given positive integer $\kappa$.

For each $j \in\{1, \ldots, N\}$, let $\mathbb{Y}_{j}$ denote all the wireless signals observed at the $3 M$ antennas of BS $j$ :

$$
\mathbb{Y}_{j}:=\left\{\left(\mathbf{Y}_{u, 1}, \ldots, \mathbf{Y}_{u, n}\right): \text { sector } u \text { is in cell } j\right\}
$$

and let $\mathbb{V}_{\text {to } j}^{k-1}$ denote the $6 \cdot(k-1)$ cooperation messages BS $j$ has received from its 6 neighboring BSs during the first $k-1$ cooperation rounds:

$$
\begin{array}{r}
\mathbb{V}_{\text {to } j}^{k-1}:=\left\{V_{i^{\prime} \rightarrow j}^{k^{\prime}}: \text { BS } i^{\prime} \text { adjacent to BS } j\right. \\
\text { and } \left.k^{\prime}=1, \ldots, k-1\right\} .
\end{array}
$$


In each cooperation round $k \in\{1, \ldots, \kappa\}$, each BS $j \in\{1, \ldots, N\}$ calculates the message $V_{j \rightarrow i}^{k}$ that it sends to a neighboring $\mathrm{BS} i$ as:

$$
V_{j \rightarrow i}^{k}=\psi_{j \rightarrow i}^{k,(n)}\left(\mathbb{Y}_{j}, \mathbb{V}_{\text {to } j}^{k-1}\right)
$$

for some cooperation functions $\left\{\psi_{j \rightarrow i}^{k,(n)}\right\}$ on appropriate domains. The cooperation functions have to be chosen so that for each pair of neighbouring BSs $i, j \in\{1, \ldots, N\}$, the total information a BS $j$ sends over the backhaul link to BS $i$ does not exceed rate $\mu>0$ :

$$
\frac{1}{n} \sum_{k=1}^{\kappa} H\left(V_{j \rightarrow i}^{k}\right) \leq \mu
$$

Once the BS-cooperation phase is terminated, each BS $j \in\{1, \ldots, N\}$ proceeds to decode the three messages sent by the mobiles in the three sectors of its cell:

$$
\mathbf{W}_{j}:=\left\{W_{u}: \text { sector } u \text { is in cell } j\right\}
$$

To this end, it applies a decoding function $h_{j}$ on corresponding domains to produce the message estimates

$$
\hat{\mathbf{W}}_{j}:=h_{j}\left(\mathbb{Y}_{j}, \mathbb{V}_{\text {to } j}^{\kappa}\right)
$$

An error occurs in the communication unless for each cell $j \in\{1, \ldots, N\}$ :

$$
\hat{\mathbf{W}}_{j}=\mathbf{W}_{j}
$$

or equivalently, unless for each sector $u \in\{1, \ldots, 3 N\}$ :

$$
\hat{W}_{u}=W_{u}
$$

\section{Sum-Capacity and Degrees of Freedom}

A rate-tuple $\left(R_{1}, \ldots, R_{3 N}\right)$ is said to be achievable if, for every $\epsilon>0$ and sufficiently large blocklength $n$, there exist encoding, cooperation, and decoding functions $\left\{f_{u}^{(n)}\right\},\left\{\psi_{j, i}^{k,(n)}\right\}$, and $\left\{h_{j}^{(n)}\right\}$, such that

$$
\mathbb{P}\left[\bigcup_{u=1}^{3 N}\left\{\hat{W}_{u} \neq W_{u}\right\}\right] \leq \epsilon .
$$

The capacity region $\mathrm{C}(\mathrm{P}, \mu, \kappa)$ is defined as the closure of the set of all achievable rate-tuples, the sum capacity $\mathrm{C}_{\Sigma}(\mathrm{P}, \mu, \kappa)$ is defined as

$$
\mathrm{C}_{\Sigma}(P, \mu, \kappa):=\sup _{\substack{\left(R_{1}, \ldots, R_{3 N}\right) \\ \in \mathrm{C}(P, \mu, \kappa)}} \sum_{u \in\{1, \ldots, 3 N\}} R_{u}
$$


and the average per-user capacity $\overline{\mathrm{C}}(P, \mu, \kappa)$ is defined as

$$
\overline{\mathrm{C}}(P, \mu, \kappa):=\frac{\mathrm{C}_{\Sigma}(P, \mu, \kappa)}{3 N} .
$$

In the high signal-to-noise ratio (SNR) regime, the quantity of interest is the degrees of freedom $(D o F)$, which is defined as:

$$
\operatorname{DoF}\left(\mu_{\text {DoF }}, \kappa\right):=\lim _{N \rightarrow \infty} \lim _{P \rightarrow \infty} \frac{\overline{\mathrm{C}}\left(P, \mu_{\text {DoF }} \cdot \frac{1}{2} \log P, \kappa\right)}{\frac{1}{2} \log P} .
$$

Notice that here the backhaul cooperation rate $\mu$ scales with the power constraint $P$.

Both the sum capacity and the DoF of our system depend on the values of the channel matrices. In this manuscript we present bounds on the DoF that hold for all generic channel matrices [8], i.e., for a set of channel matrices that occur with probability 1 if the entries of the channel matrices are drawn independently of each other according to any continuous distribution.

\section{UplinK SCHEME WiTh COOPERATIVE BSs}

The main contribution of this paper is a new uplink communication scheme with BS cooperation. In this scheme, some of the mobile users are deactivated, i.e., transmit no messages, and the other mobile users use multi-antenna Gaussian codebooks to send their own messages. (To attain fairness among users, different versions with different sets of deactivated mobile users can be time-shared.) Receiving BSs quantize their output signals and send the resulting quantization messages to specific BSs, which we call master BSs, where each master BS jointly decodes the subset of messages corresponding to the quantized output signals it received. After this decoding step, the master BSs send back the decoded messages to the BSs to which the messages are actually intended. The main novelty in our work is a strategy to deactivate users so as to decompose the network into non-interfering clusters while keeping more users active than in the naive approach.

The details of the scheme are as described in the following, where the positive integer $\gamma$ denotes the single parameter of the scheme. Choose the master BSs so that they build a regular grid of equilateral triangles where the three master cells forming each of the triangles are lying $2 \gamma$ cell-hops apart from each other. As we will see, the proposed scheme takes place over $2 \gamma$ cooperation rounds, and thus one is allowed to choose any

$$
\gamma \in\left\{1, \ldots,\left\lfloor\frac{\kappa}{2}\right\rfloor\right\} .
$$

For simplicity, we first present the scheme in the special case $\gamma=2$. The general case is treated later. 


\section{A. Special case $\gamma=2$}

Consider the network in Figure 2, where the magenta cells indicate the master cells. We refer to cells that are adjacent to master cells as layer-1 cells, and the cells that are adjacent to layer-1 cells and are neither master cells nor layer-1 cells as layer-2 cells. We choose the master cells in such a way that every cell is either a master cell, a layer-1 cell or a layer-2 cell.

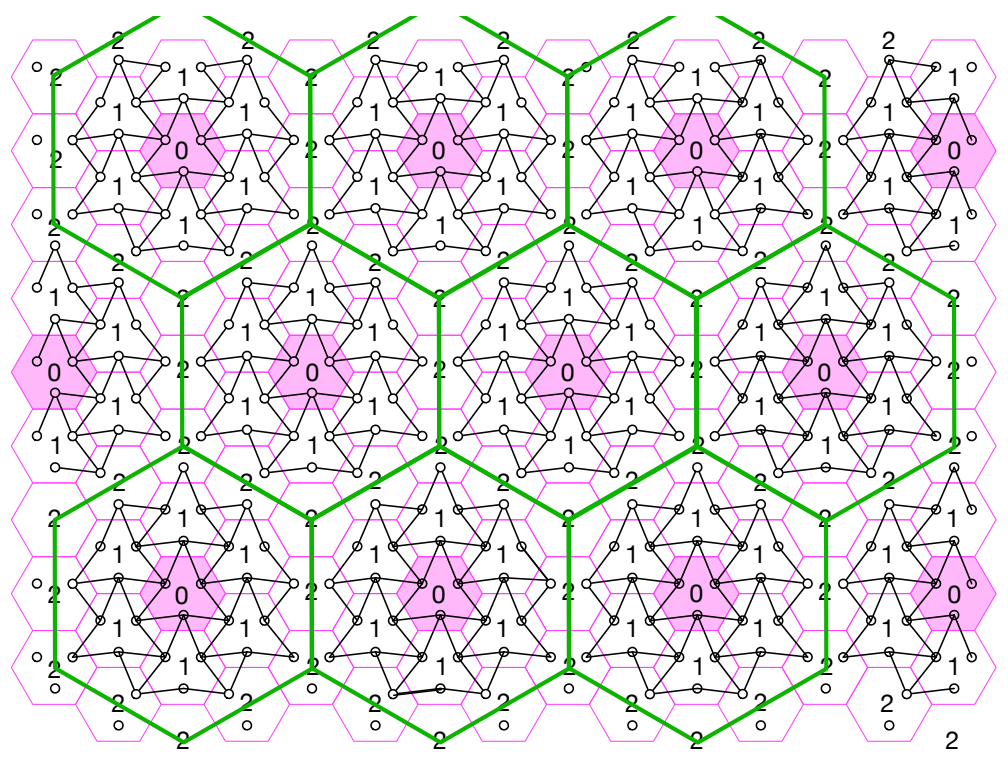

Fig. 2: Illustration of our uplink coding scheme with BS cooperation for parameter $\gamma=2$. Magenta BSs decode all the messages sent by mobile users in the decoding regions defined by the green hexagons and then pass the decoded messages to their intended receiving BSs.

Encoding: The mobile users in the network that are not shown in Figure 2 remain silent and do not transmit anything. Each of the remaining mobile users $u$ transmits its Message $M_{u}$ using an independent Gaussian codebook of power $P$.1

Cooperation between BSs: Cooperation takes place over $2 \gamma=4$ rounds as follows:

- Round 1: Each layer-2 BS applies an independent rate- $R_{q}$ (a parameter to be determined later) MIMO-Gaussian vector quantizer to the $M$-dimensional receive signal of each of its sectors with an active mobile user. It sends the quantization message obtained for a given

\footnotetext{
${ }^{1}$ In order to satisfy the block-power constraint in 2, the codebook needs to be generated with a power $P^{\prime}$ that is slightly smaller than $P$. The codewords such generated that do not satisfy 2 are discarded. This is however a technicality that we ignore.
} 
sector to one of the adjacent layer-1 BSs that lie in the same green hexagonal region as this sector, see Figure 2 .

Each layer-1 BS applies a rate- $R_{q}$ MIMO-Gaussian vector quantizer to its own $3 \mathrm{M}$ dimensional receive signals, and it sends the 3 quantization messages to the adjacent master cell.

- Round 2: The layer-1 BSs forward the Round-1 messages they received from layer-2 BSs to their adjacent master BSs.

- Rounds 3 and 4: Each master BS uses the quantization messages received in Rounds 1 and 2 to reconstruct quantized versions of the $27 M$-dimensional received signals observed in the sectors lying in the same green hexagonal region as the master BS itself. Using these 27 quantized $M$-dimensional signals and its own $3 M$-dimensional receive signals, each master BS decodes all the messages sent by the 30 active mobile users in the same green hexagonal region as the master BS itself.

In Rounds 3 and 4, messages decoded at the master BS but intended for layer-1 or layer-2 BSs are sent over the backhaul links to the intended BSs. Specifically, messages for layer-1 BSs are directly passed from the master BSs to their intended BSs in Round 3. Messages for layer-2 BSs are first passed to adjacent layer-1 BSs in Round 3, and then to the intended layer-2 BSs in Round 4.

Decoding: Each master BS declares the message that it has produced during the decoding steps preceding cooperation-round 3. Each layer-1 BS declares the messages it has received during the cooperation-round 3 and that were intended for it. Each layer-2 BS declares the messages that it has received during the round-cooperation 4 .

DoF Analysis: For clarity here we limit ourselves to a DoF analysis. The interest here is in the high-SNR asymptotics, i.e., on the degrees of freedom. Choosing the quantization-rate

$$
R_{q}=\frac{M}{2} \log (P)
$$

ensures that each received signal is essentially quantized at the noise level and allows to achieve the same DoF as if the master BS directly had access to all the 30 receive signals in its decoding region. Since the channel matrix corresponding to the active sectors in a single green hexagonal region is of full rank with probability 1, and by the symmetry of the problem, each of these 30 transmitted messages can be decoded with arbitrarily small probability of error at a DoF of $M$ 
per active user. Notice now that for large networks $(N \rightarrow \infty)$ a fraction of $\frac{5}{6}$ mobile users are active, and thus the scheme achieves a DoF of

$$
\operatorname{DoF}_{\gamma}=M \frac{5}{6}, \quad \gamma=2
$$

We analyze the backhaul load for a single green hexagonal region. In Round 1, layer-2 BSs send in total 9 quantization messages, each of $M \mathrm{DoF}$, to layer-1 BSs, and layer-1 BSs send in total 18 quantization messages, each of $M$ DoF, to the adjacent master BS. In Round 2, layer-1 BSs send in total 9 quantization messages, each of $M$ DoF, to the adjacent master BS. In Round 3, the master BS sends 27 decoded messages, each of DoF $M$, to the 6 adjacent layer-1 BSs. From these 27 decoded messages, 9 are forwarded to adjacent layer-2 BSs during cooperation-round 4 . So, in total 72 cooperation messages of DoF $M$ are sent in each green hexagonal region.

The backhaul load in our scheme is unevenly distributed among backhaul links. To balance this load and to achieve fairness among the rates achieved by the various mobile users, instances of the presented scheme are time-shared, where in different instances different cells play the role of master cells. We are then interested in the average backhaul DoF occupied by our scheme.

To calculate this average backhaul DoF, notice that each green hexagonal region is associated with a single master cell, and that for $N \gg 1$ a cell partitioning can be obtained by associating 11 neighboring cells to each of the master cells as illustrated in Figure 3. (Note that the green hexagonal regions in Figure 2 do not represent a valid cell partitioning because some cells are associated with multiple master cells.) Including the master cell, each subset of the cell partitioning thus consists of 12 cells. Notice further that each cell is surrounded by 6 neighbouring cells and has 6 outgoing backhaul links. The average backhaul DoF per cooperation link is obtained by dividing the total backhaul DoF occupied in a single hexagonal region, i.e., $72 M$, by the total number of backhaul links associated to a single subset of the cell partitioning, i.e., $6 \cdot 12$ :

$$
\mu_{\mathrm{DoF}, \gamma}=\frac{72 M}{6 \cdot 12}=M, \quad \gamma=2
$$

\section{B. General case $\gamma>0$}

Choose the master cells so that they form a regular pattern of equilateral triangles where the master cells building these triangles are $2 \gamma$ cell-hops apart from each other. See Figure 4 for a choice of magenta master cells when $\gamma=3$. For $\ell=1, \ldots, \gamma$, we call layer- $\ell$ cells, the cells that 


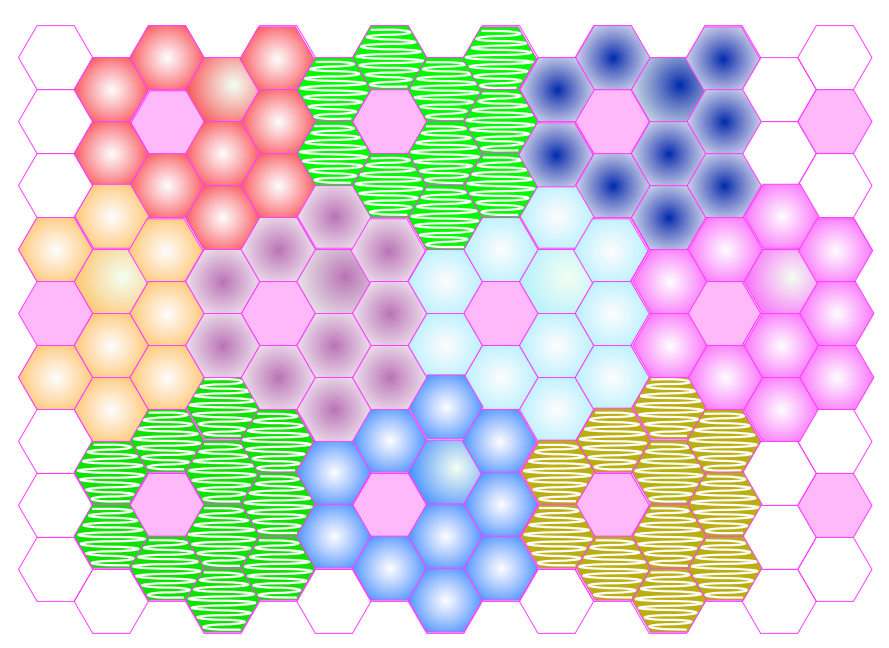

Fig. 3: The figure illustrates the proposed cell partitioning. The subsets of 11 cells associated to different master cells are shown in different colors and different shadings. (Master cells are in pink color.)

are at distance $\ell$ from the closest master cell. The distance between two cells here is defined as the minimum number of cell-hops needed to get from one cell to the other.

Encoding: Some of the layer- $\gamma$ mobile users are deactivated and do not transmit any message at all. All other mobile users are active, and send their messages using an independent Gaussian codebook of power $P$. To describe the mobile users that are deactivated, consider the layer- $\gamma$ cells that are adjacent to 3 layer- $(\gamma-1)$ cells. These cells will be called the corner cells. For every second of these corner cells, all the mobile users are deactivated; for all other corner cells, all mobile users are kept active. More specifically, for any two corner cells that are closest to each other, for one of them all users are deactivated and for the other one all users are active. For all other layer- $\gamma$ cells, the single mobile user is deactivated that is closest to a corner cell with only active users. So, among all the layer- $\gamma$ cells surrounding a master cell, 3 of them have only active users, 3 have only deactivated users, and the remaining $6(\gamma-1)$ have two active users. See Figure 4 for an example when $\gamma=3$.

Cooperation between BSs: Cooperation takes place over $2 \gamma$ rounds.

- Rounds 1 to $\gamma$ : Each BS that is not a master BS applies a rate- $R_{q}$ (a parameter to be determined later) MIMO-Gaussian vector quantizer to any of its $M$-dimensional receive 


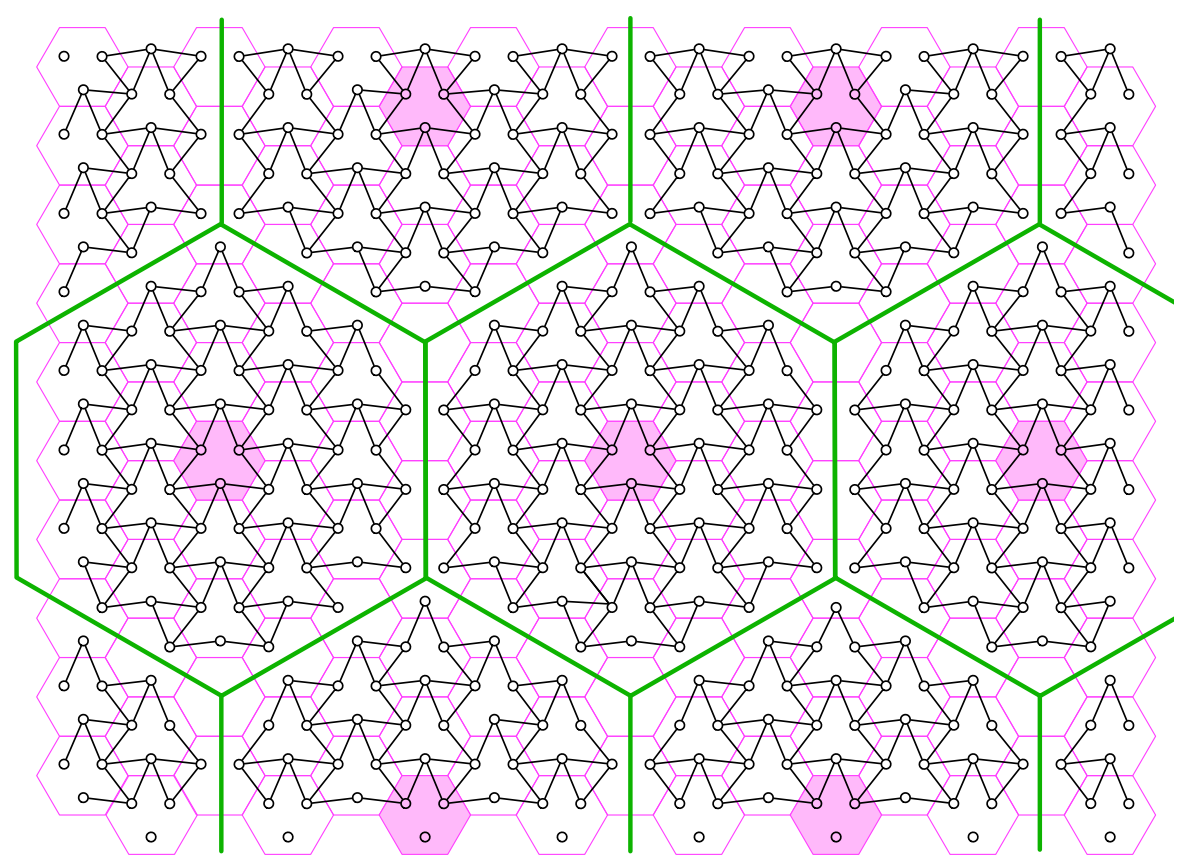

Fig. 4: Illustration of our uplink coding scheme with BS cooperation for parameter $\gamma=3$. Magenta BSs decode all the messages sent by mobile users in the decoding regions defined by the green hexagons and then pass the decoded messages to their intended receiving BSs.

signals that correspond to a sector with an active mobile user. For $\ell=1, \ldots, \gamma-1$, each layer $\ell$ BS sends its 3 quantization messages to the closest master BS, by means of multihop communication over $\ell$ rounds. Each layer- $\gamma$ BS sends each of its produced quantization messages to the master BS that is closest to the sector of each of the quantized signals. This transmission is again multi-hop over $\gamma$ rounds.

- Rounds $\gamma+1, \ldots, 2 \gamma$ : Each master BS reconstructs the $M$-dimensional quantized signals corresponding to the quantization messages received in Rounds $1, \ldots, \gamma$. It then decodes the messages sent by the mobile users in the sectors corresponding to these quantization messages as well as the messages sent by the mobile users in its own cell. Finally, during cooperation rounds $\gamma+1, \ldots, 2 \gamma$, it communicates each of the decoded messages using multi-hop communication to its intended BS.

Decoding: Each master BS directly declares the messages guessed for its own cell before cooperation round $\gamma+1$. The other BSs declare the messages that the master BSs have guessed for their cells and which they have learned during cooperation rounds $\gamma+1, \ldots, 2 \gamma$. 
DoF Analysis: For clarity we again start with a DoF analysis. As before, the quantization rate $R_{q}$ is chosen as in (13). This choice allows us to achieve the same DoF as if the master BSs directly had access to all the receive signals for which they receive quantization information. Each BS in the first $\gamma-1$ layers sends three quantization messages (one for each sector) to the master cell; three of the BSs in layer $\gamma$ send no quantization information at all; and the remaining BSs in layer $\gamma$ send a single quantization message. Since each layer $\ell=1,2, \ldots$ contains $6 \ell$ cells, each master BS receives in total

$$
3 \cdot \sum_{\ell=1}^{\gamma-1} 6 \ell+(6 \gamma-3)=9 \gamma(\gamma-1)+6 \gamma-3=3\left(3 \gamma^{2}-\gamma-1\right)
$$

quantization messages. Each such quantization message describes an $M$-dimensional receive signal. With these quantization messages and the $3 M$-dimensional receive signals observed in its own cell, a master cell decodes the messages of $3 \gamma(3 \gamma-1)$ mobile users. Since each mobile user is equipped with $M$ transmit antennas and since the master cell applies joint decoding as in a single-receiver MIMO multi-access scenario, each of the considered mobile users can reliably transmit its message with DoF $M$.

To calculate the average per-user DoF over the entire network, we describe a cell partitioning that associates each cell to a unique master cell, and that associates the same number of cells to each master cell. (Note that Fig. 4 does not describe a valid partition, because some cells are associated with more than one master cell.) The average per-user DoF is then obtained by dividing the DoF achieved at a given master cell, i.e., $M \cdot 3 \gamma(3 \gamma-1)$, by the total number of sectors that are contained in a single subset of the proposed cell partitioning. For $N \gg 1$ the desired cell partitioning can be obtained by associating to each master BS a set of sectors that includes all its layer-1,,$\gamma-1$ cells and the layer- $\gamma$ cells that lie on its north-east, east, and south-east. Hereby, the corner cells in the north and the south are excluded. (See Figure 5.)

Each subset of the described cell partitioning thus includes a master cell, all its layer-1, $2, \ldots, \gamma-$ 1 cells, and half of its layer- $\gamma$ cells but one. It's total number of cells is thus:

$$
1+\sum_{\ell=1}^{\gamma-1} 6 \ell+(3 \gamma-1)=3 \gamma^{2}
$$

and it includes $3 \cdot 3 \gamma^{2}$ sectors. We conclude that the proposed scheme achieves an average per-user DoF of

$$
\operatorname{DoF}_{\gamma}=\frac{M \cdot 3 \gamma(3 \gamma-1)}{3 \cdot 3 \gamma^{2}}=M \frac{3 \gamma-1}{3 \gamma}, \quad \gamma \in\left\{1, \ldots,\left\lfloor\frac{\kappa}{2}\right\rfloor\right\}
$$




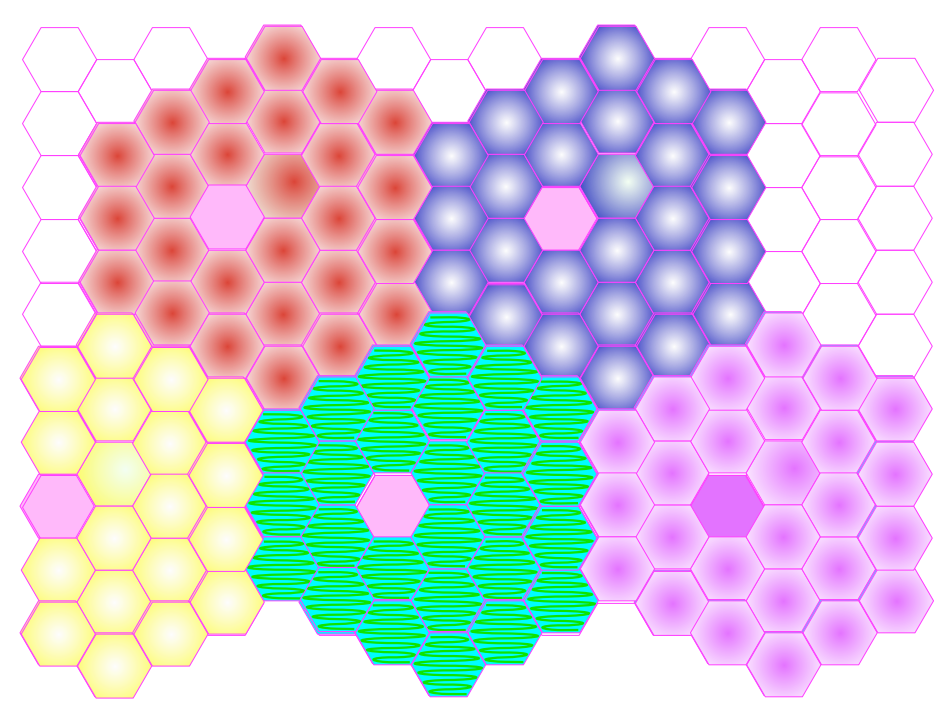

Fig. 5: The figure illustrates the proposed cell partitioning when $\gamma=3$. Specifically, the pink cells indicate the master cells and different colors and shadings are used to indicate the subsets of cells associated to the different master cells.

We next analyze the backhaul load that is associated with a single master cell. During Rounds $1, \ldots, \gamma$, a master BS receives a quantization message of DoF $M$ for each of the sectors that have an active user and that are closer to this master BS than to any other master BS. Since the quantization message for a sector in layer- $\ell$ is communicated over $\ell$ backhaul links, the total backhaul DoF on these rounds is

$$
\begin{aligned}
& 3 M \sum_{\ell=1}^{\gamma-1} 6 \ell \cdot \ell+M(6 \gamma-3) \gamma \\
& =3 M[(\gamma-1) \gamma(2 \gamma-1)+(2 \gamma-1) \gamma] \\
& =3 M \gamma^{2}(2 \gamma-1) .
\end{aligned}
$$

During Rounds $\gamma+1, \ldots, 2 \gamma$ a decoded message of DoF $M$, is communicated from the master BS to the BSs for each quantization message that has been sent during Rounds $1, \ldots, \gamma$. Therefore, the total backhaul load during Rounds $1, \ldots \gamma$ coincides with the total backhaul load during Rounds $\gamma+1, \ldots, 2 \gamma$, and the total backhaul load over all rounds is thus

$$
6 M \gamma^{2}(2 \gamma-1)
$$

To balance the backhaul load and to achieve fairness among the rates achieved by the various mobile users, instances of the presented scheme are time-shared, where in different instances 
different cells play the role of the master cells. Under such a time-sharing framework, the average backhaul DoF is the limiting quantity. Consider again the cell partitioning proposed above to calculate the DoF. The average backhaul DoF is obtained by dividing (20) by the number of outgoing (or incoming) backhaul links pertaining to a single subset of the cell partitioning, which by (17) is $6 \cdot 3 \gamma^{2}$ :

$$
\mu_{\mathrm{DoF}, \gamma}=\frac{2 \cdot 3 M \gamma^{2}(2 \gamma-1)}{6 \cdot 3 \gamma^{2}}=M \frac{2 \gamma-1}{3}, \quad \gamma \in\left\{1, \ldots,\left\lfloor\frac{\kappa}{2}\right\rfloor\right\} .
$$

Analysis of Average Per-User Rate: We now analyze the scheme at finite SNR, where for simplicity we restrict to $M=1$ antenna. For a given parameter $\gamma$, we choose the quantization rate as

$$
R_{q}:=\frac{3}{2 \gamma-1} \mu
$$

By similar arguments to (21), this choice ensures that the backhaul rate-limitation (6) is satisfied. We assume that the BSs adjust their quantizers to the channel realization. Then, if $\mathbf{h}_{u}$ denotes the channel vector from all users to the receive antenna in sector $u$, the above chosen rate implies the the quantized signal in this sector $u$ has an quantization noise variance of:

$$
\sigma_{q, u}^{2}=\frac{P\left\|\mathbf{h}_{u}\right\|^{2}+1}{2^{2 R_{q}}-1}=\frac{P\left\|\mathbf{h}_{u}\right\|^{2}+1}{2^{\frac{6}{2 \gamma-1} \mu}-1} .
$$

Since the quantization noises are independent across antennas, and there are $3 \gamma^{2}$ cells in a cluseter, the average per-user achievable rate is characterized as

$$
R_{\text {ach }}=\frac{1}{18 \gamma^{2}} \cdot \log \frac{\left|P \mathrm{H}_{\gamma} \mathrm{H}_{\gamma}^{\top}+\Sigma_{q, \gamma}+\mathrm{I}\right|}{\left|\Sigma_{q, \gamma}+\mathrm{I}\right|},
$$

where $\Sigma_{q, \gamma}$ denotes the diagonal matrix with entry $\sigma_{q, u}^{2}$ in the row and column that correspond to sector $u$.

We numerically compare this rate to the rates of the following three schemes:

- Scheme 1 is a variation of our proposed scheme, but no users are silenced and interference from other clusters is treated as noise. The previously silenced users now also apply power$P$ Gaussian codebooks and their messages are decoded by the master cells of their clusters as explained before. Notice that the previously silenced users lie on the borders of different clusters, and one can therefore freely choose with which cluster to associate them. We propose to use the regular "wind-spinner clusters" assignment described in our patent [6], 
where each cluster is assigned the same number of "previously-deactivated" users. The comparison with our proposed scheme allows to identify whether at finite SNR users should be deactivated or not.

- Scheme 2 is a naive variation of our proposed scheme where for a given parameter $\gamma$, all layer- $\gamma$ cells are deactivated. The admissible values for the parameter $\gamma$ are thus $\gamma=$ $1, \ldots,\left\lfloor\frac{\kappa}{2}\right\rfloor+1$. (Notice that the choice $\gamma=1$ splits the networks into isolated cells and the scheme does not exploit the cooperation links at all.)

- Scheme 3 is inspired by current practical systems. The decoding depends on the realization of the channel coefficients. With the help of its neighbors, each BS identifies for each user in its cell the 3 adjacent sectors that give the best joint decoding performance for the corresponding message. It then collects the (quantized versions of) these $3 M$ output signals, and based on them decodes the desired messages. Notice that this scheme only involves one collaboration step and hence can be implemented for any positive cooperation complexity $\kappa \geq 1$.

In our numerical comparison, we average the rate over 5000 realizations of the channel matrices, where for each realization all channel gains are drawn independently of each other according to a Gaussian distribution. The direct channel gains of intra-sector links are drawn with variance 1 and the cross channel gains of inter-sector links are drawn with variance $\alpha$, where $\alpha$ models the path attenuation. The backhaul capacity is assumed to grow with power $P$ as $\mu=\mu_{\text {DoF }} \cdot \frac{1}{2} \log (1+P)$, for a given backhaul DoF $\mu_{\mathrm{DoF}}>0$. Figure 6 shows the average rates of the different schemes as a function of the power $P$ in $\mathrm{dB}$ for different choices of the path loss factor $\alpha \in\{0.16,0.5\}$, the backhaul DoF $\mu_{\mathrm{DoF}} \in\{0.5,1,2.5\}$, and the maximum allowed cooperation complexity $\kappa \in\{2,4\}$. When $\kappa=4$, two different choices for the parameter $\gamma$ are possible. For the scheme proposed in this paper and the wind-spinner cluster scheme in [6] (Scheme 1) the choices $\gamma=1$ or $\gamma=2$ are allowed. Notice that for a given quantization rate, it is always beneficial to choose $\gamma$ as large as possible because it increases the benefit of cooperation. However, the larger $\gamma$, the more backhaul capacity is required. For a fixed backhaul capacity it can thus be beneficial to choose a smaller $\gamma$ which allows to increase the quantization rate and thus the quality of the master BS's observations. Our numerical results reveal that for $\mu_{\mathrm{DoF}}=0.5$ and $\alpha=0.16$ in both schemes it is optimal to choose parameter $\gamma=1$ at all considered power levels. For a larger backhaul DoF, e.g., $\mu_{\mathrm{DoF}}=1$, in the newly proposed scheme the choice $\gamma=1$ is optimal for powers 
below $13 \mathrm{~dB}$ and in the wind-spinner scheme of [6] it is optimal for powers below $10 \mathrm{~dB}$. For larger powers the choice $\gamma=2$ performs better. For the naive scheme that silences entire cells (Scheme 2), parameters $\gamma=1,2,3$ are possible when $\kappa=4$. When $\alpha=0.16$ for both $\mu_{\mathrm{DoF}}=0.5$ and $\mu_{\mathrm{DoF}}=1$, it is optimal to choose parameter $\gamma=2$ at all power levels. For $\alpha=0.16$ and $\mu_{\mathrm{DoF}}=2.5$, large quantization rates are available for all choices of $\gamma$, and as a consequence this parameter should be chosen as large as possible in all schemes and at almost all power levels (except for $P=0$ or $P=1 \mathrm{~dB}$ ). Similar observations hold also under a strong interference assumption that we model as $\alpha=0.5$.

Comparing Figures $6 \mathrm{a}-6 \mathrm{c}$, we observe that when $\alpha=0.16$ and $\kappa=4$, the opportunistic BSselection scheme (Scheme 3) outperforms the others at small power levels. At higher power levels however the performance of Scheme 3 significantly degrades compared to the other schemes. For large backhaul capacity, the wind-spinner clustering scheme (Scheme 1) outperforms the others at moderate powers. The scheme proposed in this paper outperforms all other schemes for sufficiently large powers, irrespectively of the available backhaul capacity. From Figures 6d and $6 \mathrm{e}$ we observe that under strong interference, $\alpha=0.5$, the proposed scheme outperforms all other schemes at all power levels even when backhaul capacity is moderate and even when only two cooperation rounds are permitted.

\section{LOWER AND UPPER BOUNDS ON DOF}

We present lower and upper bounds on the DoF for generic [8] channel coefficients, where the coefficients are drawn independently of each other from a continuous distribution.

Define $\mu_{\mathrm{DoF}, 0}=0$ and $\operatorname{DoF}_{0}=\frac{M}{3}$, and for each $\gamma \in\left\{1, \ldots,\left\lfloor\frac{\kappa}{2}\right\rfloor\right\}$ :

$$
\begin{aligned}
\mu_{\mathrm{DoF}, \gamma} & :=M \frac{2 \gamma-1}{3} \\
\mathrm{DoF}_{\gamma} & :=M\left(1-\frac{1}{3 \gamma}\right) .
\end{aligned}
$$

Theorem 1 (Achievability): For generic channel coefficients, the DoF is lower bounded as

$$
\operatorname{DoF}\left(\mu_{\mathrm{DoF}}, \kappa\right) \geq \operatorname{upp} \text { conv hull }\left\{\left(\mu_{\mathrm{DoF}, \gamma}, \operatorname{DoF}_{\gamma}\right): \gamma=0, \ldots,\left\lfloor\frac{\kappa}{2}\right\rfloor\right\}
$$

Proof: The claim follows by time-sharing the scheme proposed in Section III for different values of $\gamma$. 
Figure 7 illustrates this lower bound for different values of $\kappa$. Notice that even with only two conferencing rounds, a DoF of $M / 2$ is achievable with our scheme with a backhaul DoF of only $\mu_{\mathrm{DoF}}=0.5$.

For comparison, notice that Schemes 1 and 3 presented at the end of the previous section are not interesting in the high-SNR regime because the processed signals are interfered by the signals from neighboring clusters. More specifically, the wind-spinner clustering approach cannot get a DoF larger than the scheme proposed in this paper, because the signals of the additionally activated cells all suffer from out-of-cluster interference. (The wind-spinner clustering scheme moreover requires higher backhaul DoF than our new scheme because more signals are quantized and sent to the master cell.) Even worse, the practical opportunistic BS-selection scheme typically does not achieve any positive DoF, because all signals are interfered from other signals sent from mobiles in other cells.

We compare the DoF of our new scheme to the DoF of the naive clustering scheme (Scheme 2) in the previous section. Recall that unlike in our proposed scheme, in the naive clustering approach the parameter $\gamma$ can be chosen as any integer value between 1 and $\left\lfloor\frac{\kappa}{2}\right\rfloor+1$. Following similar steps as in the analysis of our new scheme, it can be shown that for any fixed $\gamma \in$ $\left\{1,2, \ldots,\left\lfloor\frac{\kappa}{2}\right\rfloor+1\right\}$, the naive scheme achieves the backhaul-rate DoF pair:

$$
\begin{aligned}
\mu_{\text {naive }, \gamma} & :=M \frac{(2 \gamma-1)(\gamma-1)}{3} \\
\text { DoF }_{\text {naive }, \gamma} & :=M\left(1-\frac{3 \gamma-1}{3 \gamma^{2}}\right)
\end{aligned}
$$

The DoF achieved by this naive scheme (Scheme 2) is thus equal to

$$
\operatorname{DoF}_{\text {naive }}=\text { upp conv hull }\left\{\left(\mu_{\text {naive }, \gamma}, \text { DoF }_{\text {naive }, \gamma}\right): \gamma=1,2, \ldots,\left\lfloor\frac{\kappa}{2}\right\rfloor+1\right\}
$$

Figure 8 compares the new lower bound in Theorem 1 with the lower bound achieved by the naive approach. The figure also shows the information-theoretic upper bound stated below. Notice that the lower bounds on the DoF are based on practically implementable coding schemes. In particular, classical interference alignment is not used, even though it is known to achieve a DoF of $M / 2$ over a generic network in the absence of BS cooperation. In contrast, the informationtheoretic upper bound holds for any possible coding scheme, obviously including interference alignment. It is thus natural that the presented upper and lower bounds match only approximately. 
Theorem 2 (Converse): The DoF is upper bounded as

$$
\operatorname{DoF}\left(\mu_{\mathrm{DoF}}, \kappa\right) \leq \min \left\{\frac{M}{2}+\frac{2}{3} \mu_{\mathrm{DoF}}, M\left(1-\frac{1}{2\left(1+\kappa+\kappa^{2}\right)}\right)\right\} .
$$

Proof: See Appendix A

\section{CONCLUDING REMARKS}

The paper presents upper and lower bounds on the DoF of the uplink of a sectorized cellular network with backhaul cooperation between BSs. A main feature of this paper is that this DoF is characterized in function of the backhaul rate and the maximum number of allowed cooperation rounds. The presented results show that limiting the number of cooperation rounds also limits the DoF achievable over the network. In particular, by Theorem 2, to get a DoF of $M$ an infinite number of cooperation rounds is required as the number of cells in the network grows. When the number of cooperation rounds does not grow with the number of the cells but is bounded, then also the DoF is bounded away from 1, even in the idealized case of unlimited backhaul cooperation rate.

The upper bound on the DoF is information-theoretic and applies to any coding scheme. The lower bound is based on a simple practically implementable scheme: the transmitting mobiles either remain silent or use Gaussian point-to-point codes; receiving BSs apply vector quantizers to their $M$-dimensional receive signals and send the quantization messages over the backhaul links to so called master BSs which decode a cluster of messages based on its own receive signals and the quantization signals it receives over the backhaul links. No advanced tools such as interference alignment are required in this scheme. The main novelty of the scheme is the proposed choice of which mobiles remain silent and which transmit their messages. In fact, this choice exploits the sectorization of the network and is done in a way that only few users remain silent but the network still decomposes into non-interfering clusters. The decomposition ensures that interference is not propagated across clusters and therefore facilitates decoding at the BSs.

The scheme is shown to not only attain an improved DoF, but also improved average-per user rate in the finite SNR regime.

It is straightforward to obtain the dual results also for the downlink. To this end, BSs should first send messages to their closest master cells, which then jointly encodes all its available messages, i.e., it pre-calculates the transmit signals at the close-by BSs. These transmit signals are quantized and distributed over the backhaul links to their respective BSs, which in their 
turn send these quantized signals over the network. Mobile users apply simple point-to-point reception techniques. This however does not limit their achievable DoF if the master cell has well encoded the messages, i.e., has chosen a good precoding matrix.

\section{APPENDIX A \\ PROOF OF THEOREM 2}

We extend [21, Lemma 1] to this hexagonal model with receiver-conferencing between adjacent BSs but without transmitter-conferencing. We start by proving the upper bound

$$
\operatorname{DoF}\left(\mu_{\mathrm{DoF}}, \kappa\right) \leq M \cdot\left(\frac{1}{2}+\frac{2}{3} \mu_{\mathrm{DoF}}\right) .
$$

To this end, partition the cells of the network alternately into red and white cells as depicted in Figure 9. Then, define

$$
\begin{aligned}
\mathbb{W}_{\text {red }} & :=\left\{W_{u}: u \text { is a mobile user in a red cell }\right\} \\
\mathbb{X}_{\text {red }} & :=\left\{\mathbf{X}_{u}: u \text { is a mobile user in a red cell }\right\} \\
\mathbb{Y}_{\text {red }} & :=\left\{\mathbb{Y}_{i}: i \text { is a red cell }\right\} \\
\mathbb{Y}_{\text {white }} & :=\left\{\mathbb{Y}_{i}: i \text { is a white cell }\right\}
\end{aligned}
$$

as well as for each round $k=1, \ldots, \kappa$, the conferencing messages

$$
\begin{aligned}
\mathbb{V}_{\text {white } \rightarrow \text { red }}^{k} & :=\left\{V_{i \rightarrow j}^{k,(n)}: i \text { is a white cell, } j \text { is a red cell }\right\} \\
\mathbb{V}_{\text {red } \rightarrow \text { red }}^{k} & :=\left\{V_{i \rightarrow j}^{k,(n)}: i, j \text { are red cells }\right\} . \\
\mathbb{V}_{\text {red } \rightarrow \text { white }}^{k} & :=\left\{V_{i \rightarrow j}^{k,(n)}: i \text { is a red cell, } j \text { is a white cell }\right\} \\
\mathbb{V}_{\text {white } \rightarrow \text { white }}^{k} & :=\left\{V_{i \rightarrow j}^{k,(n)}: i, j \text { are white cells }\right\} .
\end{aligned}
$$

To prove the converse, fix a sequence of encoding, cooperation, and decoding functions $\left\{f_{u}^{(n)}\right\}$, $\left\{\psi_{j, i}^{k,(n)}\right\}$, and $\left\{h_{j}^{(n)}\right\}$ so that for sufficiently large blocklength $n$ the probability of error does not exceed $\epsilon>0$. We show in the following that a super-receiver observing the three items listed in the following, can decode all $3 N$ messages $\left\{W_{u}\right\}$ correctly whenever the $N$ BSs decode them correctly in the original setup. The super-receiver's probability of error can thus not be larger than the original probability of error, which was upper bounded by $\epsilon$. The three items are:

1) all output signals in red cells, $\mathbb{Y}_{\text {red }}$;

2) all conferencing messages from white to red cells, $\mathbb{V}_{\text {white } \rightarrow \text { red }}^{1}, \ldots, \mathbb{V}_{\text {white } \rightarrow \text { red }}^{\kappa_{\max }}$; and 
3) some genie-information $\mathbb{G}$ that will be explained with more details later on and satisfies

$$
\lim _{P \rightarrow \infty} \frac{I\left(W_{1}, \ldots, W_{3 N} ; \mathbb{G} \mid \mathbb{Y}_{\text {red }}, \mathbb{V}_{\text {white } \rightarrow \text { red }}^{1}, \ldots, \mathbb{V}_{\text {white } \rightarrow \text { red }}^{\kappa}\right)}{\log P}=0
$$

irrespectively of the encoding, cooperation, and decoding functions.

The super-receiver decodes the messages $\left\{W_{u}\right\}_{u=1}^{3 N}$ by means of the following six decoding steps:

1) For each round $k=1, \ldots, \kappa$, it applies the appropriate cooperation functions $\left\{\psi_{j, i}^{k,(n)}\right\}$ to the red output signals $\mathbb{Y}_{\text {red }}$ and the previous rounds' conferencing messages $\left\{\mathbb{V}_{\text {white } \rightarrow \text { red }}^{k^{\prime}}\right\}_{k^{\prime}=1}^{k-1}$ and $\left\{\mathbb{V}_{\text {red } \rightarrow \text { red }}^{k^{\prime}}\right\}_{k^{\prime}=1}^{k-1}$. This allows the super-receiver to compute the conferencing messages of the current round $\mathbb{V}_{\text {red } \rightarrow \text { red }}^{k}$. (Notice that knowledge of output signals in white cells or conferencing messages sent to white cells are not required for this computation.)

2) It applies the appropriate decoding functions $\left\{h_{j}^{(n)}\right\}$ to the output signals $\mathbb{Y}_{\text {red }}$ and the conferencing messages $\left\{\mathbb{V}_{\text {red } \rightarrow \text { red }}^{k}\right\}_{k^{\prime}=1}^{\kappa}$ to decode messages $\mathbb{W}_{\text {red }}$.

3) It applies the encoding functions $\left\{f_{u}^{(n)}\right\}$ to the previously decoded messages $\mathbb{W}_{\text {red }}$ to construct input signals $\mathbb{X}_{\text {red }}$.

4) With the input and output signals sent and received in red cells, $\mathbb{X}_{\text {red }}$ and $\mathbb{Y}_{\text {red }}$, and an appropriately chosen genie-information $\mathbb{G}$, it reconstructs the output signals received at the BSs in the white cells, i.e., it reconstructs $\mathbb{Y}_{\text {white }}$. To see that this is possible, notice the following. Let $\tilde{H}_{\text {white } \rightarrow \text { white }}, \tilde{H}_{\text {red } \rightarrow \text { red }}$, and $\tilde{H}_{\text {red } \rightarrow \text { white }}$ denote the channel matrices from inputs in white cells to outputs in white cells, from inputs in red cells to outputs in red cells, and from inputs in red cells to outputs in white cells, respectively. Notice that because there are as many white as red cells, because of the regular interference structure, and because the channel coefficients are drawn independently from a continuous distribution, these matrices are squared and of full rank with probability 1. For an appropriate choice of the genie-information $\mathbb{G}$ that satisfies $(33)$, the outputs in the white cells can be constructed as:

$$
\begin{aligned}
\mathbb{Y}_{\text {white }}= & \tilde{\mathrm{H}}_{\text {white } \rightarrow \text { white }}^{-1}\left(\mathbb{Y}_{\text {red }}-\tilde{\mathrm{H}}_{\text {red } \rightarrow \text { red }} \mathbb{X}_{\text {red }}\right) \\
& +\tilde{\mathrm{H}}_{\text {red } \rightarrow \text { white }} \mathbb{X}_{\text {red }}+\mathbb{G}
\end{aligned}
$$

5) For each round $k=1, \ldots, \kappa$, it applies the appropriate cooperation functions $\left\{\psi_{j, i}^{k,(n)}\right\}$ to the white and red output signals $\mathbb{Y}_{\text {white }}$ and $\mathbb{Y}_{\text {red }}$ and to the observed and previously calculated conferencing messages $\left\{\mathbb{V}_{\text {white } \rightarrow \text { red }}^{k^{\prime}}\right\}_{k^{\prime}=1}^{k-1}$ and $\left\{\mathbb{V}_{\text {red } \rightarrow \text { white }}^{k^{\prime}}\right\}_{k^{\prime}=1}^{k-1},\left\{\mathbb{V}_{\text {red } \rightarrow \text { red }}^{k^{\prime}}\right\}_{k^{\prime}=1}^{k-1},\left\{\mathbb{V}_{\text {white } \rightarrow \text { white }}^{k^{\prime}}\right\}_{k^{\prime}=1}^{k-1}$, to compute the conferencing messages of the current round $\mathbb{V}_{\text {red } \rightarrow \text { white }}^{k}$ and $\mathbb{V}_{\text {white } \rightarrow \text { white }}^{k}$. 
6) It applies the appropriate decoding functions $\left\{h_{j}^{(n)}\right\}$ to the output signals $\mathbb{Y}_{\text {white }}$ and the calculated conferencing messages $\left\{\mathbb{V}_{\text {red } \rightarrow \text { white }}^{k}\right\}_{k^{\prime}=1}^{\kappa}$ and $\left\{\mathbb{V}_{\text {white } \rightarrow \text { white }}^{k}\right\}_{k^{\prime}=1}^{\kappa}$ so as to decode messages $\mathbb{W}_{\text {white }}$.

The above arguments imply that any tuple $\left(R_{1}, \ldots, R_{3 N}\right)$ that is achievable over the original network from mobile users to BSs is also achievable over the network from mobile users to the super-receiver. So, by Fano's inequality:

$$
\begin{aligned}
3 N R \leq & \frac{1}{n} I\left(W_{1}, \ldots, W_{3 N} ; \mathbb{Y}_{\text {red }}, \mathbb{V}_{\text {white } \rightarrow \text { red }}^{1}, \ldots, \mathbb{V}_{\text {white } \rightarrow \text { red }}^{\kappa}, \mathbb{G}\right)+\epsilon \\
= & \frac{1}{n} I\left(W_{1}, \ldots, W_{3 N} ; \mathbb{Y}_{\text {red }}\right)+\frac{1}{n} I\left(W_{1}, \ldots, W_{3 N} ;, \mathbb{V}_{\text {white } \rightarrow \text { red }}^{1} \ldots, \mathbb{V}_{\text {white } \rightarrow \text { red }}^{\kappa} \mid \mathbb{Y}_{\text {red }}\right) \\
& +\frac{1}{n} I\left(W_{1}, \ldots, W_{3 N} ; \mathbb{G} \mid \mathbb{Y}_{\text {red }}, \mathbb{V}_{\text {white } \rightarrow \text { red }}^{1}, \ldots, \mathbb{V}_{\text {white } \rightarrow \text { red }}^{\kappa}\right)+\epsilon,
\end{aligned}
$$

where $\epsilon$ tends to zero as $n \rightarrow \infty$. We notice that the first summand in $(36)$ has DoF at most $3 M\left|\mathcal{I}_{\text {red }}\right|$, where

$$
\mathcal{I}_{\text {red }}:=\{i: \text { cell } i \text { is } \operatorname{red}\}
$$

the second summand in (36) has DoF at most $4\left|\mathcal{I}_{\text {white }}\right| \cdot \mu_{\text {DoF }}$, where

$$
\mathcal{I}_{\text {white }}:=\{i: \text { cell } i \text { is white }\}
$$

and the third summand in (36) has zero DoF by assumption (33). Dividing (36) by $\log P$ and taking the limit $P \rightarrow \infty$, we obtain the following bound on the DoF:

$$
3 N \cdot \operatorname{DoF} \leq 3 M\left|\mathcal{I}_{\text {red }}\right|+4\left|\mathcal{I}_{\text {white }}\right| \cdot \mu_{\text {DoF }}
$$

Dividing the above by $3 N$ and letting $N \rightarrow \infty$ establishes the desired bound after noticing that

$$
\varlimsup_{N \rightarrow \infty} \frac{\left|\mathcal{I}_{\text {red }}\right|}{N}=\varlimsup_{N \rightarrow \infty} \frac{\left|\mathcal{I}_{\text {white }}\right|}{N}=1 / 2 .
$$

We now prove the upper bound

$$
\operatorname{DoF}\left(\mu_{\mathrm{DoF}}, \kappa\right) \leq M \cdot\left(1-\frac{1}{2+\kappa+\kappa^{2}}\right) .
$$

We partition the set of cells $\{1, \ldots, N\}$ into red, blue, and white cells. For $\kappa=2$ and $\kappa=3$ the cell partitioning is illustrated in Figures 10 and 11 . In general, the partitioning is obtained by choosing red and white cells so that they form the corner points of a regular pattern of equilateral triangles with corner points that are $\kappa$ cell-hops apart from each other and have different colors (so two are red and one is white or two are white and one is red). The cell partitioning also has to ensure that there is the same number of white and red cells. The remaining cells are blue. 
Define the sets

$$
\begin{aligned}
\mathcal{I}_{\text {red }} & :=\{i: \text { cell } i \text { is red }\} \\
\mathcal{I}_{\text {white }} & :=\{i: \text { cell } i \text { is white }\} \\
\mathcal{I}_{\text {blue }} & :=\{i: \text { cell } i \text { is blue }\}
\end{aligned}
$$

To determine the fractions of cells that are red, blue, and white, we notice that we can partition the set of blue cells by associating a different set of $\kappa(\kappa+1)$ blue cells to each red and to each white cell. (See the green parallelepipeds in Figures 10 and 11.) This establishes the following limiting behaviors: $:^{2}$

$$
\begin{aligned}
\lim _{N \rightarrow \infty} \frac{\left|\mathcal{I}_{\text {red }}\right|}{N} & =\frac{1}{2\left(\kappa^{2}+\kappa+1\right)}, \\
\lim _{N \rightarrow \infty} \frac{\left|\mathcal{I}_{\text {white }}\right|}{N} & =\frac{1}{2\left(\kappa^{2}+\kappa+1\right)}, \\
\lim _{N \rightarrow \infty} \frac{\left|\mathcal{I}_{\text {blue }}\right|}{N} & =\frac{\kappa^{2}+\kappa}{\kappa^{2}+\kappa+1} .
\end{aligned}
$$

Define

$$
\begin{aligned}
\mathbb{W}_{\text {red }} & :=\left\{W_{u}: u \text { is a mobile user in a red cell }\right\} \\
\mathbb{X}_{\text {red }} & :=\left\{\mathbf{X}_{u}: u \text { is a mobile user in a red cell }\right\} \\
\mathbb{Y}_{\text {red }} & :=\left\{\mathbb{Y}_{i}: i \text { is a red cell }\right\} \\
\mathbb{Y}_{\text {blue }} & :=\left\{\mathbb{Y}_{i}: i \text { is a blue cell }\right\} \\
\mathbb{Y}_{\text {white }} & :=\left\{\mathbb{Y}_{i}: i \text { is a white cell }\right\}
\end{aligned}
$$

as well as for each round $k=1, \ldots, \kappa$, the conferencing messages

$$
\begin{aligned}
\mathbb{V}_{\text {blue } \rightarrow \text { red }}^{k} & :=\left\{V_{i \rightarrow j}^{k,(n)}: i \text { is a blue cell }, j \text { is a red cell }\right\} \\
\mathbb{V}_{\text {red } \rightarrow \text { blue }}^{k}: & =\left\{V_{i \rightarrow j}^{k,(n)}: i \text { is a red cell }, j \text { is a blue cell }\right\} \\
\overline{\mathbb{V}}_{\text {blue } \rightarrow \text { blue }}^{k} & :=\left\{V_{i \rightarrow j}^{k,(n)}: i, j \text { are blue cells and } i \text { is at most } \kappa-k \text { cell-hops from a red cell }\right\} .
\end{aligned}
$$

Notice that $\overline{\mathbb{V}}_{\text {blue } \rightarrow \text { blue }}^{k}$ contains only some of the round- $k$ conferencing messages sent from blue cells to blue cells. In particular, it contains only the conferencing messages that do not depend on the outputs in the white cells.

\footnotetext{
${ }^{2}$ The limit $N \rightarrow \infty$ is only needed to eliminate edge effects.
} 
To prove the converse, fix a sequence of encoding, cooperation, and decoding functions $\left\{f_{u}^{(n)}\right\}$, $\left\{\psi_{j, i}^{k,(n)}\right\}$, and $\left\{h_{j}^{(n)}\right\}$ so that for sufficiently large blocklength $n$ the probability of error does not exceed $\epsilon>0$. Notice that a super-receiver we next describe can decode all the $3 N$ messages $\left\{W_{u}\right\}_{u=1}^{3 N}$ sent by the mobile users with probability of error at most $\epsilon$. This super-receiver has access to:

- all output signals in red cells, $\mathbb{Y}_{\text {red }}$;

- all output signals in blue cells, $\mathbb{Y}_{\text {blue }}$; and

- some genie-information $\mathbb{G}$ that will be defined in more detail later on and that satisfies

$$
\lim _{P \rightarrow \infty} \frac{I\left(W_{1}, \ldots, W_{3 N} ; \mathbb{G} \mid \mathbb{Y}_{\text {red }}, \mathbb{Y}_{\text {blue }}\right)}{\log P}=0
$$

Notice that if the super-receiver performs the following six decoding steps, then it will decode all $3 N$ messages $\left\{W_{u}\right\}$ correctly whenever the $N$ BSs decode them correctly in the original setup. The super-receiver's probability of error can thus not be larger than the probability of error in the original setup.

The six decoding steps at the super-receiver are:

1) For each round $k=1, \ldots, \kappa$, it applies the appropriate cooperation functions $\left\{\psi_{j, i}^{k,(n)}\right\}$ to the output signals $\mathbb{Y}_{\text {red }}$ and $\mathbb{Y}_{\text {blue }}$ and to the previously computed conferencing messages $\left\{\mathbb{V}_{\text {red } \rightarrow \text { blue }}^{k^{\prime}}\right\}_{k^{\prime}=1}^{k-1},\left\{\mathbb{V}_{\text {blue } \rightarrow \text { red }}^{k^{\prime}}\right\}_{k^{\prime}=1}^{k-1}$, and $\left\{\overline{\mathbb{V}}_{\text {blue } \rightarrow \text { blue }}^{k^{\prime}}\right\}_{k^{\prime}=1}^{k-1}$, so as to obtain the conferencing messages of the current round $\mathbb{V}_{\text {blue } \rightarrow \text { red }}^{k}, \mathbb{V}_{\text {red } \rightarrow \text { blue }}^{k}$, and $\overline{\mathbb{V}}_{\text {blue } \rightarrow \text { blue }}^{k}$ (Notice that knowledge of output signals in white cells or conferencing messages exchanged with white cells are not required for this computation.)

2) It applies the appropriate decoding functions $\left\{h_{j}^{(n)}\right\}$ to the output signals $\mathbb{Y}_{\text {red }}$ and the conferencing messages $\left\{\overline{\mathbb{V}}_{\text {blue } \rightarrow \text { red }}^{k}\right\}_{k^{\prime}=1}^{\kappa}$ so as to decode messages $\mathbb{W}_{\text {red }}$.

3) It applies the encoding functions $\left\{f_{u}^{(n)}\right\}$ to the previously decoded messages $\mathbb{W}_{\text {red }}$ to construct input signals $\mathbb{X}_{\text {red }}$.

4) With the input and output signals sent and received in red cells, $\mathbb{X}_{\text {red }}$ and $\mathbb{Y}_{\text {red }}$, and an appropriately chosen genie-information $\mathbb{G}$, it reconstructs the output signals received at the BS in the white cells $\mathbb{Y}_{\text {white }}$

5) For each round $k=1, \ldots, \kappa$, it applies the appropriate cooperation functions $\left\{\psi_{j, i}^{k,(n)}\right\}$ to the red, white, and blue output signals $\mathbb{Y}_{\text {red }}, \mathbb{Y}_{\text {white }}$, and $\mathbb{Y}_{\text {blue }}$ and to the previously calculated conferencing messages to compute all round- $k$ conferencing messages. 
6) It applies the appropriate decoding functions $\left\{h_{j}^{(n)}\right\}$ to the output signals $\mathbb{Y}_{\text {white }}$ and $\mathbb{Y}_{\text {white }}$ and the required conferencing messages so as to decode messages $\mathbb{W}_{\text {white }}$ and $\mathbb{W}_{\text {blue }}$.

The above arguments imply that any tuple $\left(R_{1}, \ldots, R_{3 N}\right)$ that is achievable over the original network from the mobile users to the BSs is also achievable over the network from the mobile users to the super-receiver. So, by Fano's inequality:

$$
\begin{aligned}
3 N R \leq & \frac{1}{n} I\left(W_{1}, \ldots, W_{3 N} ; \mathbb{Y}_{\text {red }}, \mathbb{Y}_{\text {blue }}, \mathbb{G}\right)+\epsilon \\
= & \frac{1}{n} I\left(W_{1}, \ldots, W_{3 N} ; \mathbb{Y}_{\text {red }}, \mathbb{Y}_{\text {blue }}\right) \\
& +\frac{1}{n} I\left(W_{1}, \ldots, W_{3 N} ; \mathbb{G} \mid \mathbb{Y}_{\text {red }}, \mathbb{Y}_{\text {blue }}\right)+\epsilon
\end{aligned}
$$

where $\epsilon$ tends to zero as $n \rightarrow \infty$. Dividing the above by $\log (1+P)$ and taking the limit $P \rightarrow \infty$, we obtain the following bound on the DoF:

$$
3 N \operatorname{Do} F \leq 3 M\left(\left|\mathcal{I}_{\text {red }}\right|+\left|\mathcal{I}_{\text {blue }}\right|\right)
$$

Dividing the above by $3 N$ and letting $N \rightarrow \infty$ yields the desired bound, by (43).

\section{REFERENCES}

[1] E. Aktas, J. Evans, and S. Hanly, "Distributed decoding in a cellular multiple-access channel," IEEE Transactions on Wireless Communications, vol. 7, no. 1, pp. 241-250, Jan 2008.

[2] E. Atsan, R. Knopp, S. Diggavi, and C. Fragouli, “Towards integrating quantize-map-forward relaying into LTE," in Proc. 2012 IEEE Inform. Theory Workshop (ITW), Sep. 2012, pp. 212-216.

[3] M. N. Bacha, J. S. Evans, and S. Hanly., "On the capacity of mimo cellular networks with macrodiversity," in Proc. 2006 Australian Communication Theory Workshop, Australia, February 2006, pp. 105-109.

[4] S. I. Bross, A. Lapidoth, and M. A. Wigger, "The Gaussian MAC with conferencing encoders," in Proc. 2008 IEEE Intern. Symp. on Inf. Theory (ISIT), July 2008, pp. 2702-2706.

[5] J. Chen, U. Mitra, and D. Gesbert, "Optimal UAV relay placement for single user capacity maximization over terrain with obstacles," in Proc. 2019 IEEE 20th International Workshop on Signal Processing Advances in Wireless Communications (SPAWC), July 2019, pp. 1-5.

[6] S. Gelincik, L. Wang, and M. Wigger, "Cell sector-clustering for joint decoding of messages," Apr. 13 2018, patent application PCT/EP2018/059535.

[7] S. Gelincik, M. Wigger, and L. Wang, "DoF in sectored cellular systems with BS cooperation under a complexity constraint," in Proc. 2018 IEEE International Symposium on Wireless Communication Systems (ISWCS), Lisbon, Portugal, August 2018.

[8] S. A. Jafar, "Interference alignment a new look at signal dimensions in a communication network," Foundations and Trends in Communications and Information Theory, vol. 7, no. 1, pp. 1-134, 2011.

[9] G. Katz, B. M. Zaidel, and S. Shamai (Shitz), "On layered transmission in clustered cooperative cellular architectures," in Proc. 2013 IEEE International Symposium on Information Theory (ISIT), Istanbul, Turkey, Jul. 7-12, 2013, pp. 1162-1166.

[10] M. N. Khormuji and E. G. Larsson, "Improving collaborative transmit diversity by using constellation rearrangement," in Proc. 2007 IEEE Wireless Communications and Networking Conference, March 2007, pp. 803-807. 
[11] S.-J. Kim, S. Jain, and G. B. Giannakis, "Backhaul-constrained multi-cell cooperation using compressive sensing and spectral clustering," in Proc. 2012 IEEE 13th International Workshop on Signal Processing Advances in Wireless Communications (SPAWC), Cesme, Turkey, June 17-20 2012, pp. 65-69.

[12] G. Kramer, I. Mari, and R. D. Yates, "Cooperative communications," Foundations and Trends in Networking, vol. 1, no. 34, pp. 271-425, 2007. [Online]. Available: http://dx.doi.org/10.1561/1300000004

[13] J. N. Laneman, G. W. Wornell, and D. N. C. Tse, "An efficient protocol for realizing cooperative diversity in wireless networks," in Proc. 2001 IEEE International Symposium on Information Theory (IEEE Cat. No.01CH37252), June 2001, pp. 294-.

[14] N. Levy and S. Shamai, "Clustered local decoding for wyner-type cellular models," IEEE Transactions on Information Theory, vol. 55, no. 11, pp. 4967-4985, November 2009.

[15] V. Ntranos, M. A. Maddah-Ali, and G. Caire, "Cellular interference alignment," IEEE Transactions on Information Theory, vol. 61, no. 3, pp. 1194-1217, March 2015.

[16] A. Sanderovich, O. Somekh, H. V. Poor, and S. S. (Shitz), "Uplink macro diversity of limited backhaul cellular network," IEEE Transactions on Information Theory, vol. 55, no. 8, pp. 3457-3478, August 2009.

[17] A. Sendonaris, E. Erkip, and B. Aazhang, "User cooperation diversity, part I: System description," IEEE Transactions on Communications, vol. 51, no. 11, pp. 1927-1938, Nov 2003.

[18] — - "User cooperation diversity, part II: Implementation aspects and performance analysis," IEEE Transactions on Communications, vol. 51, no. 11, pp. 1939-1948, Nov 2003.

[19] O. Simeone, N. Levy, A. Sanderovich, O. Somekh, B. M. Zaidel, H. V. Poor, and S. Shamai (Shitz), "Cooperative wireless cellular systems: An information-theoretic view," Foundations and Trends in Communications and Information Theory, Now Publishers Inc., Hanover MA, vol. 8, no. 1-2, pp. 1-177, 2012.

[20] O. Simeone, H. V. P. O. Somekh, and S. S. (Shitz), "Local base station cooperation via finite-capacity links for the uplink of linear cellular networks,” IEEE Transactions on Information Theory, vol. 55, no. 1, pp. 190-204, January 2009.

[21] M. Wigger, R. Timo, and S. Shamai (Shitz), “Conferencing in Wyner's asymmetric interference network: Effect of number of rounds," IEEE Transactions on Information Theory, vol. 63, Mar. 2017.

[22] M. A. Wigger and G. Kramer, "Three-user MIMO MACs with cooperation," in Proc. 2009 IEEE Information Theory Workshop on Information Theory (ITW), June 2009, pp. 221-225.

[23] F. Willems, "The discrete memoryless multiple access channel with partially cooperating encoders (corresp.)," IEEE Transactions on Information Theory, vol. 29, no. 3, pp. 441-445, May 1983. 


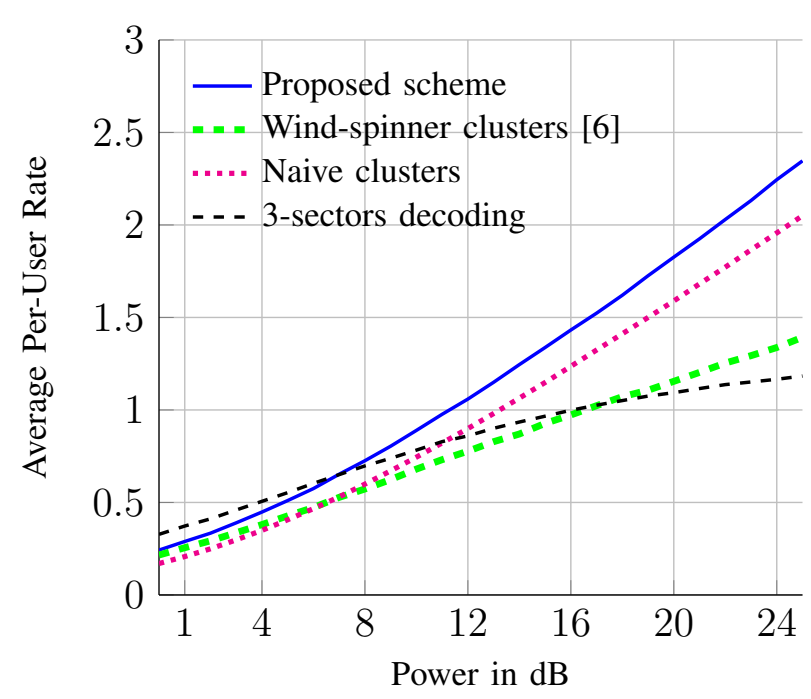

(a) Parameters $\alpha=0.16, \mu_{\mathrm{DoF}}=0.5$, and $\kappa=4$.

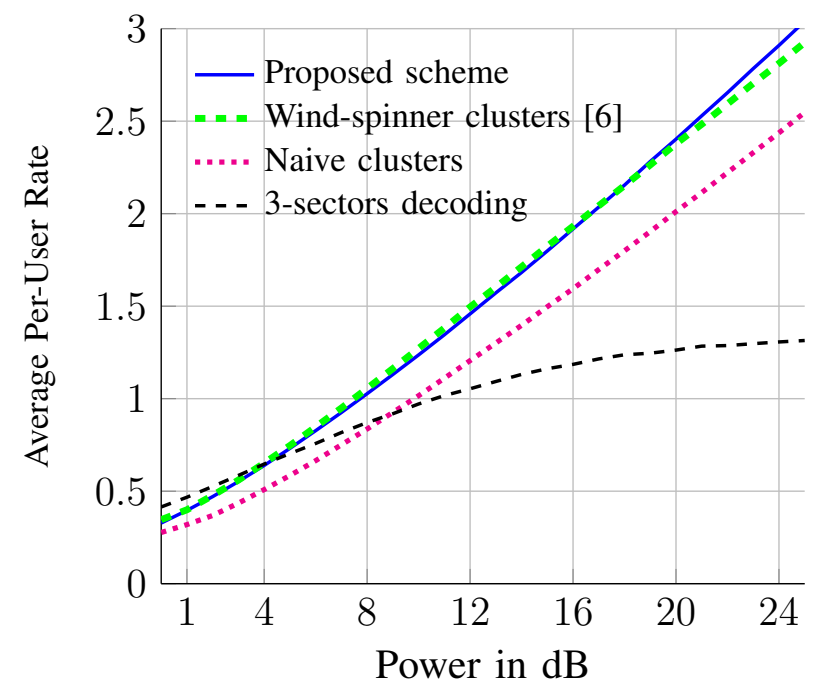

(c) Parameters $\alpha=0.16, \mu_{\mathrm{DoF}}=2.5$, and $\kappa=4$.

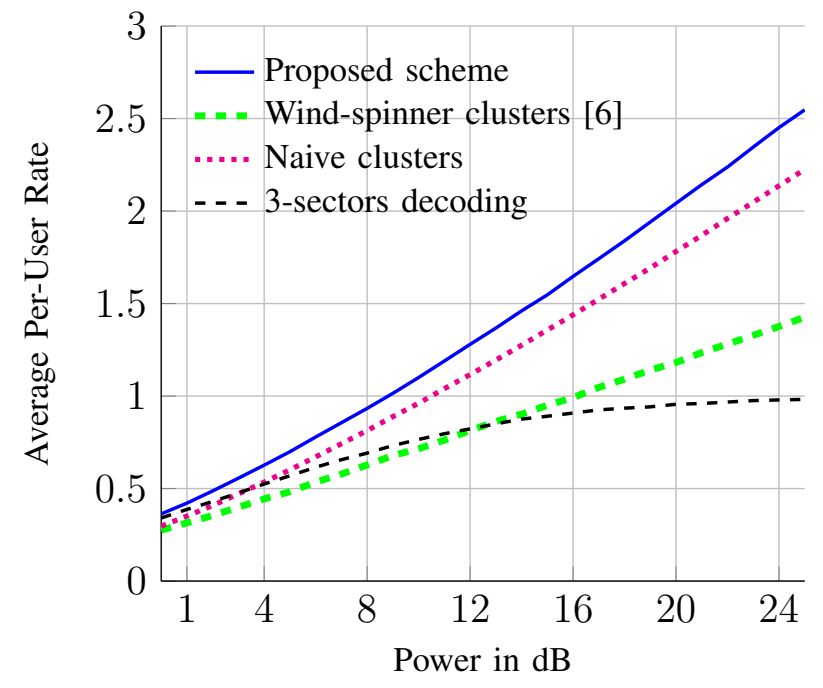

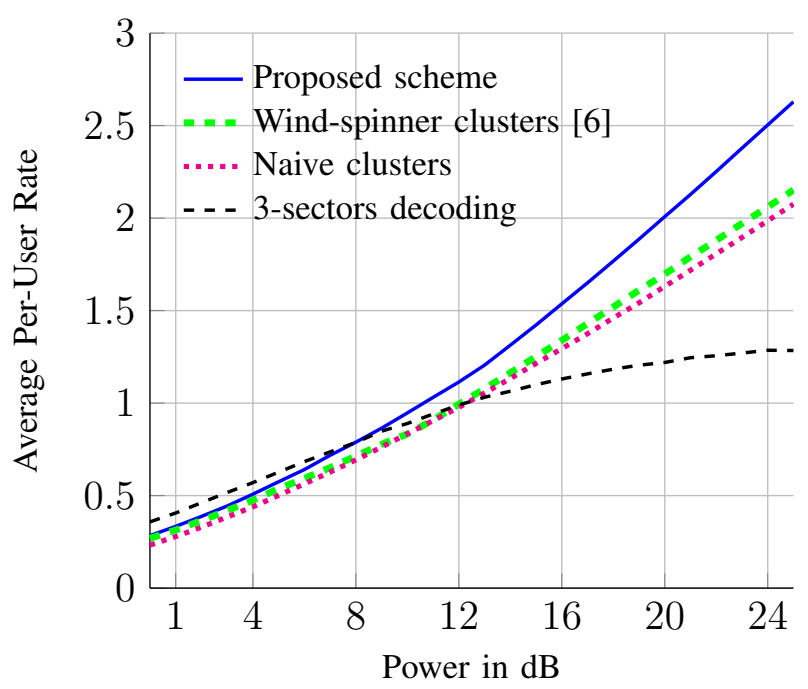

(b) Parameters $\alpha=0.16, \mu_{\mathrm{DoF}}=1$, and $\kappa=4$.

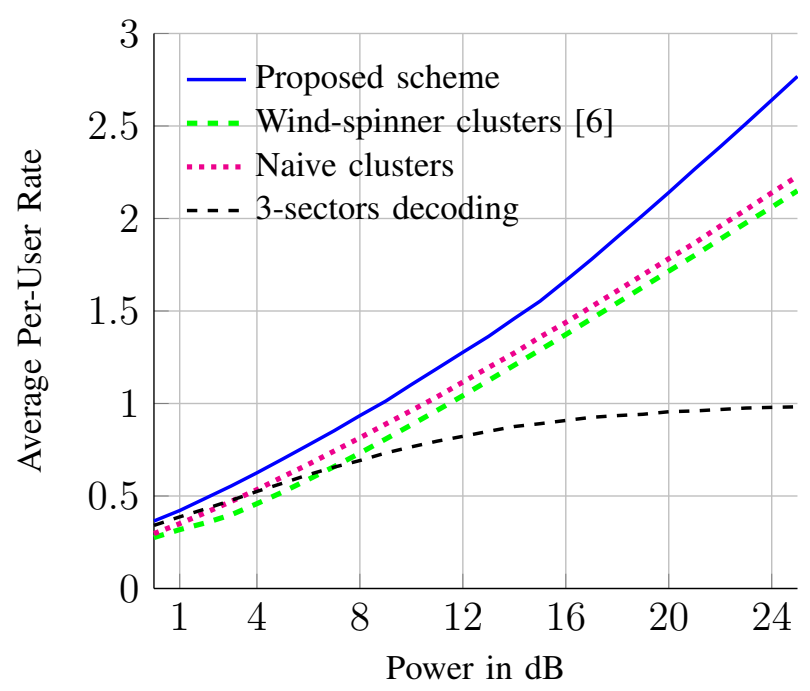

(d) Parameters $\alpha=0.5, \mu_{\mathrm{DoF}}=1$, and $\kappa=4$.

(e) Parameters $\alpha=0.5, \mu_{\mathrm{DoF}}=1$, and $\kappa=2$.

Fig. 6: Comparison of the rates achieved by the different schemes in the finite SNR regime. 


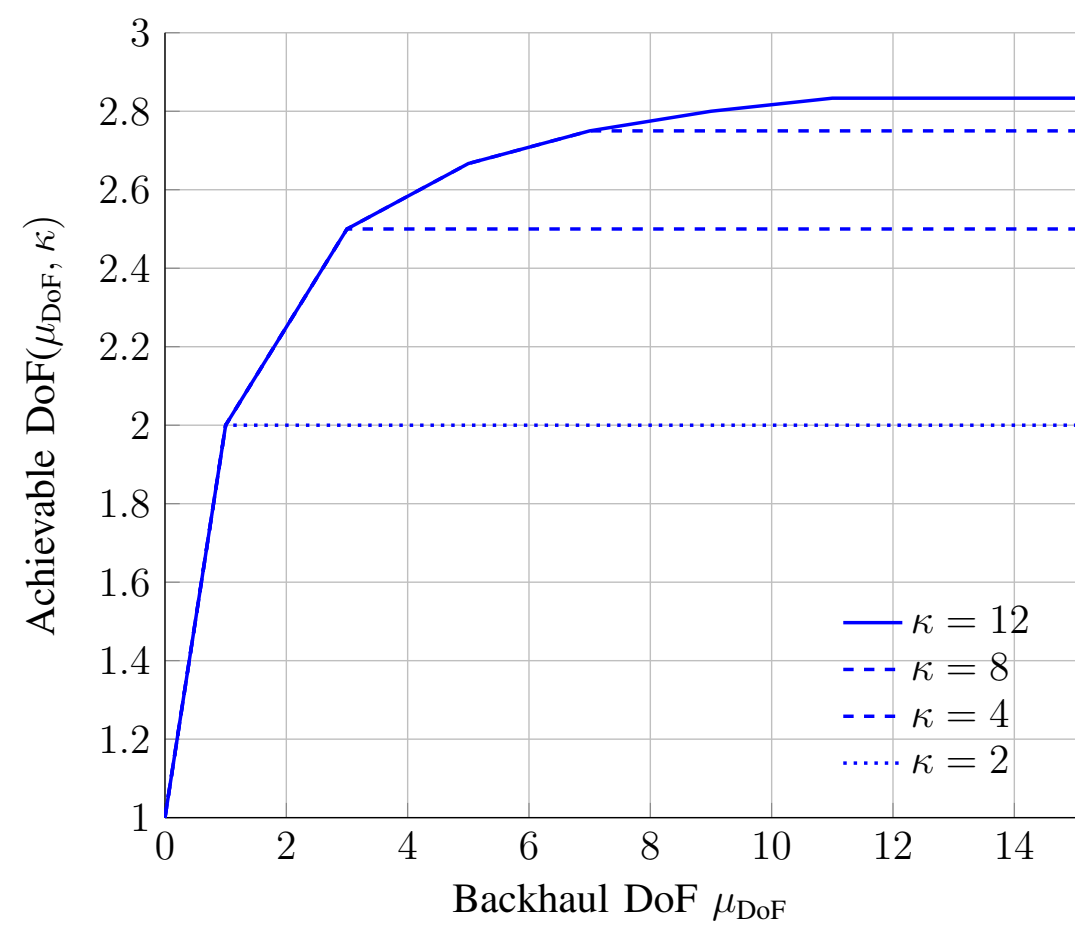

Fig. 7: Lower bound on the DoF in Theorem 1 for different values of $\kappa$ and for $M=3$, as functions of $\mu_{\mathrm{DoF}}$.
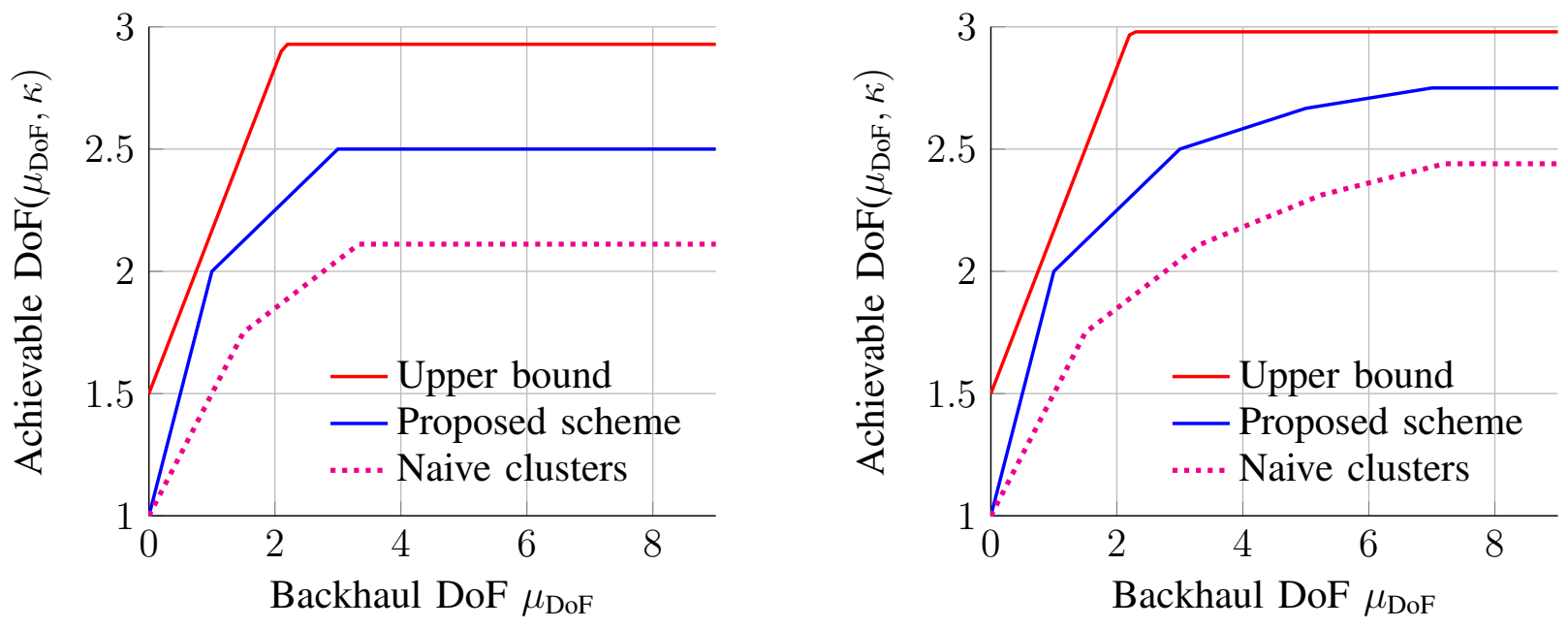

Fig. 8: Lower and upper bounds on the DoF in function of $\mu_{\mathrm{DoF}}$ for $M=3$. The figure on the left is for $\kappa=4$ and the figure on the right for $\kappa=8$. 


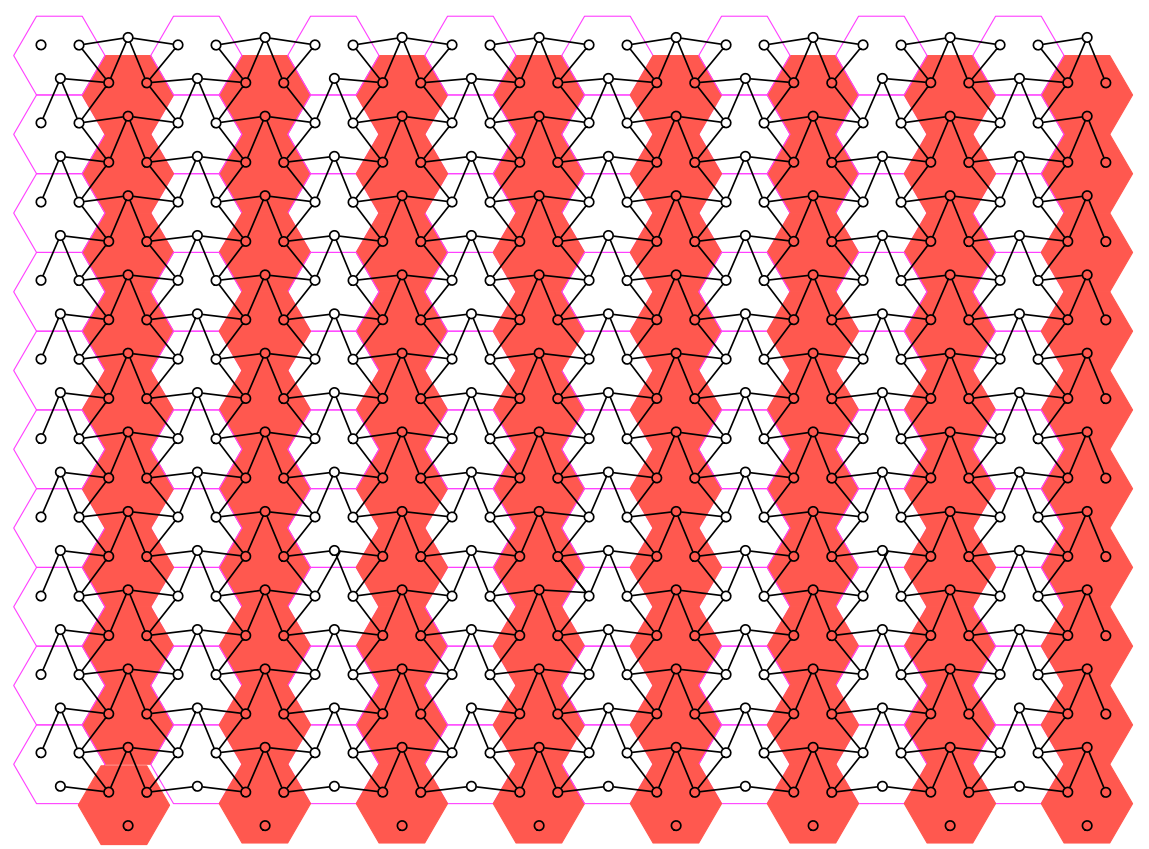

Fig. 9: Cell partitioning used for the first converse bound.

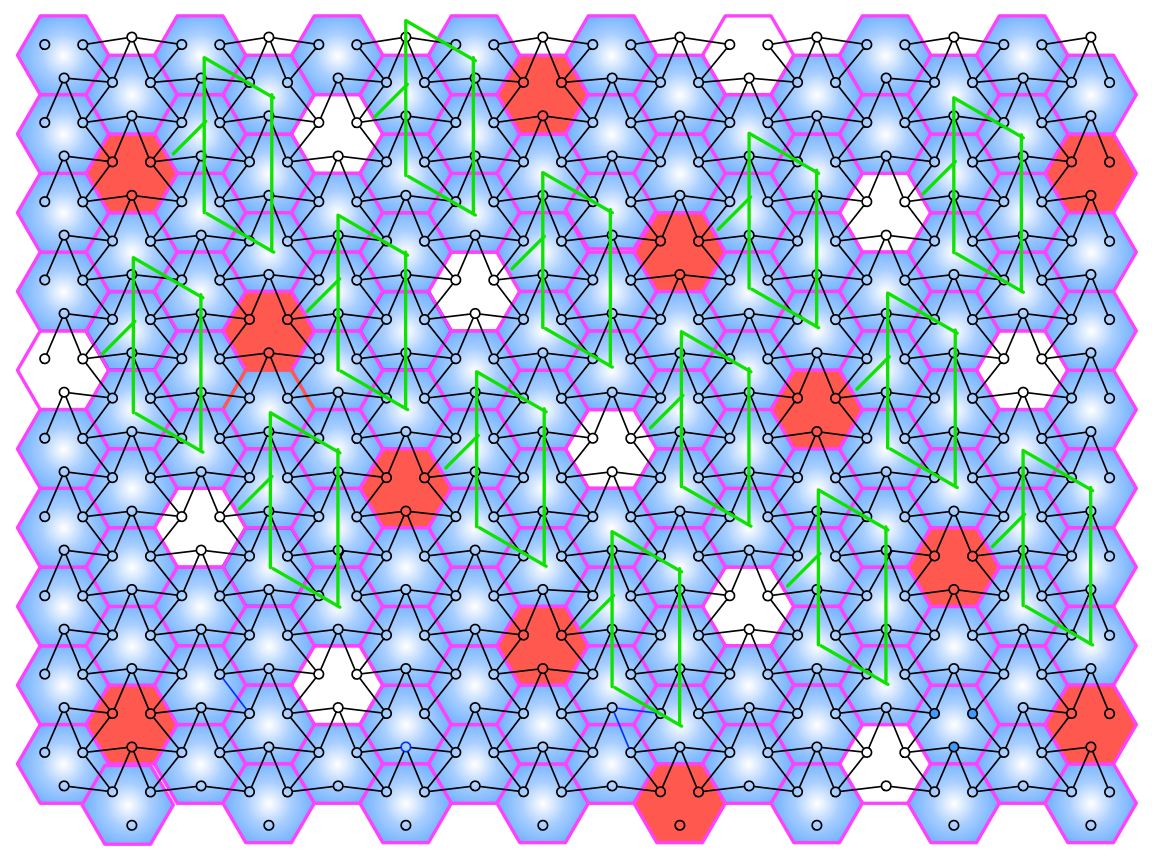

Fig. 10: Proposed cell-partitioning when $\kappa=2$. 


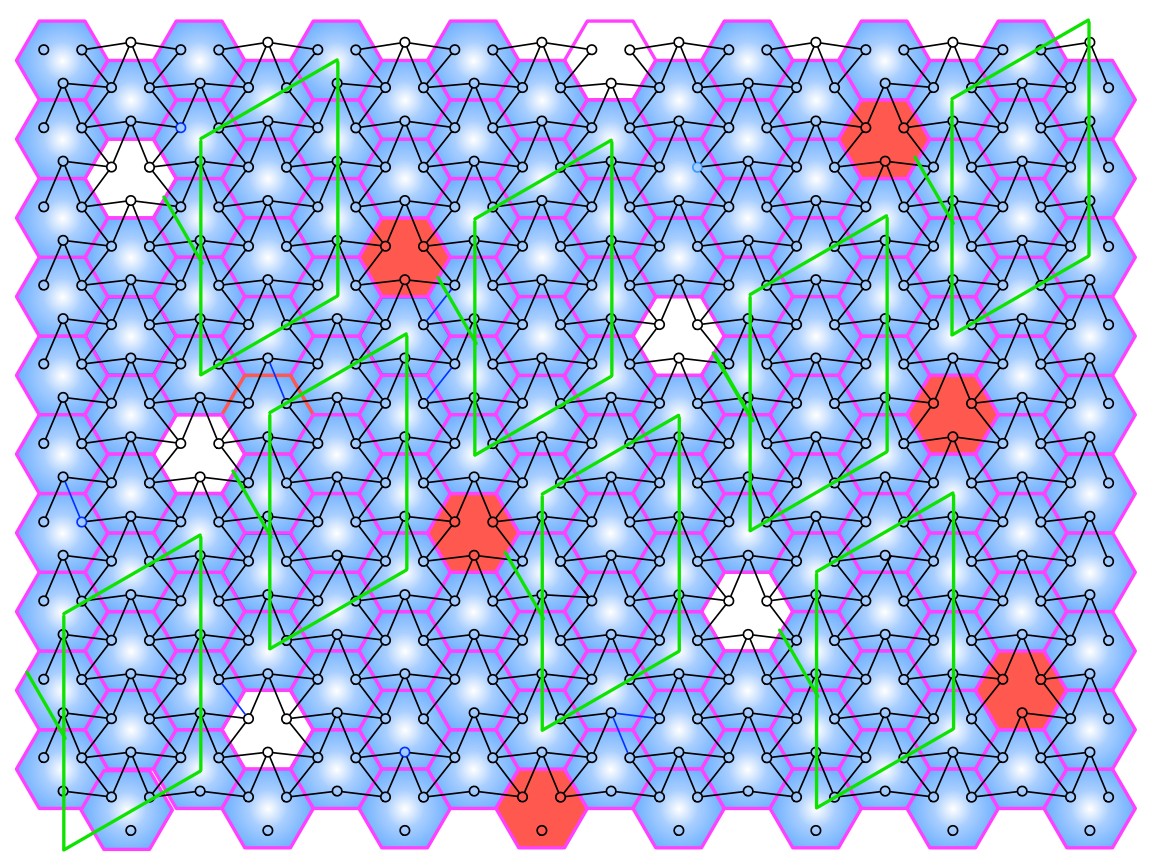

Fig. 11: Proposed cell-partitioning when $\kappa=3$. 